\title{
Plasma biomarkers for Alzheimer's Disease in relation to neuropathology and cognitive change
}

\author{
Denis S. Smirnov ${ }^{1}$. Nicholas J. Ashton ${ }^{2,3,4,5} \cdot$ Kaj Blennow $^{2,6} \cdot$ Henrik Zetterberg $^{2,6,7,8,9}$. Joel Simrén ${ }^{2,6}$. \\ Juan Lantero-Rodriguez ${ }^{2}$. Thomas K. Karikari ${ }^{2,10}$ • Annie Hiniker ${ }^{1} \cdot$ Robert A. Rissman $^{1}$ - David P. Salmon ${ }^{1}$. \\ Douglas Galasko ${ }^{1,11}$ (i)
}

Received: 5 October 2021 / Revised: 8 February 2022 / Accepted: 9 February 2022 / Published online: 23 February 2022

(c) The Author(s) 2022

\begin{abstract}
Plasma biomarkers related to amyloid, tau, and neurodegeneration (ATN) show great promise for identifying these pathological features of Alzheimer's Disease (AD) as shown by recent clinical studies and selected autopsy studies. We have evaluated ATN plasma biomarkers in a series of 312 well-characterized longitudinally followed research subjects with plasma available within 5 years or less before autopsy and examined these biomarkers in relation to a spectrum of AD and related pathologies. Plasma A $\beta 42$, A $\beta 40$, total Tau, P-tau181, P-tau231 and neurofilament light (NfL) were measured using Single molecule array (Simoa) assays. Neuropathological findings were assessed using standard research protocols. Comparing plasma biomarkers with pathology diagnoses and ratings, we found that P-tau181 (AUC $=0.856)$ and P-tau231 (AUC $=0.773)$ showed the strongest overall sensitivity and specificity for AD neuropathological change (ADNC). Plasma P-tau231 showed increases at earlier ADNC stages than other biomarkers. Plasma A $\beta 42 / 40$ was decreased in relation to amyloid and AD pathology, with modest diagnostic accuracy (AUC $=0.601)$. NfL was increased in non-AD cases and in a subset of those with ADNC. Plasma biomarkers did not show changes in Lewy body disease (LBD), hippocampal sclerosis of aging (HS) or limbic-predominant age-related TDP-43 encephalopathy (LATE) unless ADNC was present. Higher levels of P-tau181, 231 and NfL predicted faster cognitive decline, as early as 10 years prior to autopsy, even among people with normal cognition or mild cognitive impairment. These results support plasma P-tau181 and 231 as diagnostic biomarkers related to ADNC that also can help to predict future cognitive decline, even in predementia stages. Although $\mathrm{NfL}$ was not consistently increased in plasma in $\mathrm{AD}$ and shows increases in several neurological disorders, it had utility to predict cognitive decline. Plasma $\mathrm{A} \beta 42 / 40$ as measured in this study was a relatively weak predictor of amyloid pathology, and different assay methods may be needed to improve on this. Additional plasma biomarkers are needed to detect the presence and impact of LBD and LATE pathology.
\end{abstract}

Keywords Biomarker $\cdot$ Alzheimer's Disease $\cdot$ Neuropathology $\cdot$ Plasma $\cdot$ Dementia

\section{Introduction}

Pathological processes underlying Alzheimer's Disease (AD) include the aggregation of $\beta$-amyloid (A) and tau (T) proteins (forming plaques and tangles, respectively) and progressive neurodegeneration $(\mathrm{N})$, which can be combined into an ATN framework for diagnosis and staging of disease [15].

Denis S. Smirnov and Nicholas J. Ashton are the equal co-first authors.

Douglas Galasko

dgalasko@ucsd.edu

Extended author information available on the last page of the article
Exciting advances have occurred in fluid biomarkers for $\mathrm{AD}$ and related disorders-sensitive assays for protein biomarkers in plasma may enable aspects of the ATN framework to be applied in a convenient and cost-effective manner [12]. Validation studies of plasma amyloid beta 42 (A $\beta 42$ and the ratio of $A \beta 42 / A \beta 40$ ), tau, and forms of phosphorylated tau (P-tau) that were conducted primarily in relation to clinical diagnoses and positron emission tomography (PET) imaging biomarkers and/or cerebrospinal fluid (CSF) biomarkers have shown high sensitivity and specificity to detect AD-type amyloid and tau pathology [3, 17, 25, 31, 40]. Several studies have examined plasma biomarkers against neuropathology findings noted at autopsy and have found concordance between higher P-tau levels and tangle burden 
$[3,7,20,31]$; a few studies have evaluated plasma $A \beta 42$ and neurofilament light (NfL) against pathology [2, 7]. Studies to date, however, have been relatively small and have not examined biomarkers in relation to the heterogeneity of $\mathrm{AD}$ pathology. Patients with AD may vary with respect to amyloid (e.g., the degree of diffuse vs neuritic plaques; extent of cerebral amyloid angiopathy), and the co-occurrence of common neurodegenerative pathologies ( $\alpha$-synuclein and TDP-43 pathology) or vascular brain pathology.

Here, we examine the central plasma biomarkers that relate to the ATN framework in a large series of patients followed at a single research center who had neuropathologic evaluation of their brains at autopsy. We address questions of how well each biomarker distinguishes people with definite $\mathrm{AD}$ pathology from those with minimal or intermediate pathology, how these markers change longitudinally in plasma in relation to age and brain pathology, and whether co-pathology and different types of amyloid pathology influence these plasma biomarkers. Finally, we examine the relationship between plasma biomarkers and cognitive change.

\section{Materials and methods}

\section{Participants and clinical methods}

Participants were volunteers enrolled in the UCSD ShileyMarcos Alzheimer's Disease Research Center (ADRC) who underwent longitudinal annual assessments, were followed until death, and agreed to brain examination at autopsy. The research protocol was reviewed and approved by the human subject review board at the University of California, San Diego (UCSD). Informed consent was obtained from all patients or their caregivers consistent with California State law.

The comprehensive annual clinical assessment evaluates participants and uses information provided by knowledgeable informants, and includes medical and neurological history, mental status testing, assessment of psychiatric symptoms with the Neuropsychiatric Inventory (NPI), ratings of functional impairment, Clinical Dementia Rating (CDR) total score and its six subdomain scores (i.e., CDR sum of boxes), structured neurological examination, and neuropsychological assessment that is based on a comprehensive battery of cognitive tests that includes tests of global cognition (Mini-Mental State Examination [MMSE] and Dementia Rating Scale [DRS]) and tests of memory, language, attention, executive function and visuospatial abilities (at least two tests per domain) [9]. Based on each annual evaluation, participants received a research diagnosis at a consensus conference that determined an overall evaluation of cognition (normal, mild cognitive impairment [MCI; diagnosed following standard criteria], or dementia) [9]. In participants with MCI or dementia, one or more etiological diagnoses were assigned, following research criteria (e.g., AD, Dementia with Lewy Bodies [DLB], Parkinson's Disease with Dementia, Frontotemporal degeneration [FTD], other dementias). A subset of 76 subjects in our study had a research-grade brain MRI within 24 months or less of one of the plasma biomarker analyses. We analyzed the ROC for MRI imaging parameters analyzed using Freesurfer version 5.3.0 and plasma biomarkers vs ADNC at autopsy in this subgroup.

Blood was drawn from participants every 2 years unless they were unable to come to the ADRC for their evaluation. For this study, we analyzed plasma samples from subjects who had detailed neuropathological evaluation and their last blood draw 5 years or less before death. We excluded participants if they had a known dominantly inherited mutation for AD (i.e., PSEN1, PSEN2 or APP mutations), a family history of autosomal dominant $\mathrm{AD}$, or a reported age at onset (estimated from clinical interviews with the patient and an informant) younger than 50. All data (clinical and plasma) were limited to the last 10 years prior to death. For this study, baseline visit refers to the earliest blood draw (within 10 years of death) and last visit refers to the last blood draw before death. All clinic visits from baseline were used to model changes in cognition.

\section{Pathology}

Autopsy was performed using a standardized protocol [36]. Brains were divided sagittally and the left hemibrain was fixed in $10 \%$ buffered formalin while the right hemibrain was sectioned coronally and frozen at $-80{ }^{\circ} \mathrm{C}$. The formalinfixed left hemibrain was cut serially into $1 \mathrm{~cm}$ slices for paraffin embedding. Sections were taken and stained with hematoxylin and eosin (H\&E) for histopathological examination from: middle frontal cortex (Brodmann areas 8/9), rostral superior temporal cortex, inferior parietal cortex, hippocampus (CA1-CA4 and dentate gyrus), entorhinal cortex, basal ganglia, midbrain with substantia nigra, pons with locus coeruleus, and cerebellar cortex with dentate nucleus. Lesions were evaluated in $10-\mu \mathrm{m}$-thick sections stained with thioflavin-S or in 5- $\mu$ m-thick sections with immunohistochemical staining.

\section{AD pathology}

Neuritic plaques, diffuse plaques, and neurofibrillary tangles (NFT) were identified either with $1 \%$ thioflavin-S stains viewed with ultraviolet illumination and a $440 \mu \mathrm{m}$ bandpass wavelength excitation filter, or with immunohistochemical staining using antibodies to $\mathrm{A} \beta(\mathrm{Ab} 69 \mathrm{D}$, rabbit polyclonal from Edward Koo, 1:1200) and PHF1 tau (from Albert Einstein SOM (courtesy Peter Davies), 
1:600). Neuritic plaque density was estimated using methods recommended by CERAD [26], and Braak stage for NFT pathology was determined [6]. For more recent cases, pathological diagnosis of AD was made using NIA-AA consensus criteria for the postmortem diagnosis of $\mathrm{AD}$, wherein Thal phase 4-5 (A3), Braak stage V-VI (B3), and moderate-to-severe neuritic plaque density $(\mathrm{C} 2 / 3)$ corresponds to high AD neuropathologic change (ADNC) $[27,38]$. We were missing Thal phase from many cases because this evaluation was implemented only after it had been proposed as part of the NIA-AA 2012 Guidelines [27]. For consistency across older and newer cases, we, therefore, used NIA-Reagan criteria to define High ADNC versus all other categories [13]. For biomarker-pathology comparisons, we evaluated comparisons with NIA-Reagan, Braak stage and CERAD stages separately. Cerebral Amyloid Angiopathy (CAA) was graded from 0 (absent) to 3 (severe) according to procedures described by the NACC Neuropathology Working Group [27].

Non-AD pathology. Lewy body pathology identified by $\mathrm{H} \& \mathrm{E}$ staining and immunostaining with antibodies against $\alpha$-synuclein (phospho-synuclein 81A, from Virginia Lee, $1: 15,000)$ was staged according to consensus Lewy body disease (LBD) guidelines, with modification as suggested in Montine et al. to simplify the number of brain regions examined, into "brainstem", "limbic" (transitional), or "neocortical" subtypes [22]. Individuals with amygdala-predominant Lewy bodies were not included as concomitant LBD, given the low likelihood of a clinical diagnosis of LBD in this group [39]. TDP-43 pathology was identified by immunohistochemical staining (Proteintech \#10782-2-AP polyclonal, 1:12,000). Staging according to Limbic-predominant Agerelated TDP-43 Encephalopathy (LATE) consensus guidelines into "amygdala", "hippocampal", or "neocortical" stages was limited to a subset of autopsy cases processed after 2017 [29]. We expanded this series by performing immunohistochemical staining for TDP-43 on a hippocampus section on selected additional cases that allowed us to define the presence or absence of hippocampal LATE neuropathologic change to explore the association with plasma biomarkers. Hippocampal sclerosis (HS) was assessed on all cases and diagnosed when neuronal loss in the CA1 and subiculum, accompanied by gliosis, was out of proportion with the degree of AD pathology_about $80 \%$ of these cases stained positive for TDP-43 [36]. Vascular pathology was assessed by examining the brain for large arterial and lacunar infarcts, microinfarcts, and hemorrhages. Arteriolosclerosis, atherosclerosis of the circle of Willis, and amyloid angiopathy were each rated as "none", "mild", "moderate", or "severe" using a semi-quantitative 4-point scale. A Low Pathology group was defined as Braak 0-II and absence of significant LBD, major vascular pathology, LATE-NC, HS or other neurodegenerative pathology.

\section{Plasma sampling, handling and biomarker measurement}

Plasma was prepared following the UCSD Shiley-Marcos ADRC standard operating procedures. Blood was drawn from a forearm vein into EDTA citrate vacutainer tubes and centrifuged at $2000 \times g$ for $10 \mathrm{~min}$ at $4{ }^{\circ} \mathrm{C}$ in a tabletop centrifuge within $1 \mathrm{~h}$ or less of blood draw. Plasma was separated and aliquoted into $500 \mu \mathrm{L}$ fractions into polypropylene cryotubes (VWR or Sarstedt), snap frozen and stored at $-80^{\circ}$ until biomarker analyses were conducted.

Plasma biomarkers were measured using Single molecule array (Simoa) assays (Quanterix, Inc) for $A \beta 42$ and $A \beta 40$, total tau (Neurology 3-Plex A Advantage Kit \#101995), P-tau181 (pTau-181 V2 Advantage Kit \#103714), P-tau231 (University Gothenburg) [3] and NfL (NF-light ${ }^{\mathrm{TM}}$ Advantage Kit \#103186) at the Clinical Neurochemistry Laboratory, University of Gothenburg, Sweden. Across 14 analytical runs, plasma biomarkers had a repeatability $(\mathrm{A} \beta 42=9.2 \%$; $\mathrm{A} \beta 40=5.2 \%$, total tau $=3.3 \%$; P-tau181 $=7.1 \%$; P-tau231 $=5.9 \%)$ and an intermediate precision $(\mathrm{A} \beta 40=5.7 \%$, total tau $=9.2 \% ; \mathrm{P}$-tau $181=8.1 \%)$ of $<10 \%$. A $\beta 42$ and P-tau 231 had an intermediate precision of $10.8 \%$ and $12.1 \%$, respectively.

Values below assay lower limit of detection were excluded from modeling (since we did not know the true value), but were included in ROC analyses since they truly represent a low value. Values for 5 outlier samples were excluded where all biomarkers were markedly different from all other plasma draws from the same subject. Each of the subjects had at least one other plasma sample for comparison. One NfL value of $1154 \mathrm{pg} / \mathrm{mL}$ in a pathologically confirmed FTD patient was omitted from plots for visualization purposes but was retained in all statistical analyses.

\section{Statistical analysis}

Continuous demographic data were compared among pathologically confirmed groups using ANOVA followed by Tukey's HSD post hoc test if significant. Categorical demographic variables were examined using a Chi-squared test, followed by pairwise Chi-squared tests for significant results, with adjustment of $p$ values for multiple comparisons using the Benjamini and Hochberg false discovery rate method. Comparisons of biomarkers across pathologic groups or features were made using linear regressions with adjustment for age, sex, and the interval from blood draw to death. Pairwise comparisons between groups after covariate adjustment in these models were performed using the lsmeans package for $\mathrm{R}$, with $\mathrm{p}$ value adjustment for multiple comparisons by Tukey's method using the Studentized range distribution to maintain a family error rate of 0.05 . We report both unadjusted $p$ values and $p$ values adjusted 
for multiple comparisons using Tukey's method in On-line Resource tables. Only multiple comparison-adjusted values are reported in the main text and graphically displayed on figures. Receiver-operating curve (ROC) analysis was used to evaluate plasma biomarkers in relation to different degrees of AD pathology. For these ROC analyses, $95 \%$ confidence intervals for the area under the curve (AUC) were computed with 2000 stratified bootstrap replicates. Comparisons of the AUCs between biomarkers and their combinations were performed using DeLong's test. Correlations between the several CSF biomarkers were examined in various pathologically defined subgroups (e.g., AD, non-AD) using Pearson or Spearman analysis, as appropriate. Longitudinal analyses of changes of biomarkers and cognition over time used linear mixed models, with covariates added for age, sex, interval from last visit to death, as well as each variable's interaction with time. All models included random intercepts and slopes by participant. Models of cognition further included terms for the baseline performance, as well as its interaction with time. Statistical analyses were conducted with $\mathrm{R}$ version 4.0.3

\section{Results}

Demographic, clinical and APOE genotype data for 312 subjects divided into pathologically defined groups are shown in Table 1 . On average, this autopsy cohort was relatively old with a mean age at baseline $>74$ years for all groups, although the age range at baseline was wide (from 52 to 100 years; 25 th percentile 71 years and 75 th percentile 82 years). Sex distribution was relatively well balanced across groups, with a small male predominance. There was a marked over-representation of males in the Other Pathology groups (expected due to the male predominance of LBD). Mean MMSE scores were consistent with mild dementia at baseline in groups with significant AD or Other Pathology and showed progressive decline over follow-up; mean MMSE and DRS scores in the Low Pathology group showed minimal change over the follow-up interval (Table 1). APOE $\varepsilon 4$ allele frequencies were (as expected) higher in Intermediate and High ADNC groups than in Low Pathology and Other Pathology groups. The Other Pathology group primarily included patients with HS or LBD, and smaller numbers of patients with FTD, vascular pathology, or several uncommon pathologies. Table 2 shows a more detailed breakdown of neuropathology findings in the various groups. As expected, High ADNC, with or without other pathologies, was associated with the greatest severity of neuritic plaques, diffuse plaques, and CAA.

Plasma biomarkers at last blood draw for each pathologically defined subgroup are shown as box plots in Fig. 1, and summary data and detailed statistical comparisons between groups are shown in On-line Resource Tables 1 and 2. In analyses that adjusted for age, sex, and duration from last blood draw to death, the greatest differences were between High ADNC and Low Pathology for plasma P-tau181 $\left(p=9.19 \times 10^{-7}\right)$ and P-tau231 $\left(p=5.7 \times 10^{-7}\right)$. Plasma A $\beta 42$ and $\mathrm{A} \beta 42 / \mathrm{A} \beta 40(p=0.014)$ showed a decrease in patients with High ADNC vs Low Pathology. Plasma total tau and NfL were not significantly altered in relation to ADNC. Figure 1 shows that in patients with Intermediate ADNC, P-tau181 and P-tau231 showed trends for increased levels compared to those with Low Pathology (P-tau181 showed more overlap).

We examined whether levels of plasma $A \beta 42$ and $A \beta 42$ / A $\beta 40$ were associated with different forms and measures of brain amyloid pathology that were assessed with ratings of diffuse plaques, neuritic plaques (CERAD), and presence and severity of CAA. We found that plasma $\mathrm{A} \beta 42$ and $\mathrm{A} \beta 42 /$ $\mathrm{A} \beta 40$ were decreased in people with moderate or severe diffuse plaques (vs. mild diffuse plaques), and plasma $A \beta 42$ was slightly decreased in people with moderate CAA vs mild or absent CAA (Online Resource Fig. 1 and Online Resource Table 3). P-tau231 was increased in relation to severe CAA; however, severe CAA was more likely in people with higher neuritic plaque burden, and when we included neuritic plaque burden in the model, the association with CAA disappeared.

We next compared plasma biomarkers in people with various levels of ADNC defined by neuritic plaque burden, Braak stage (of tau pathology), or NIA-Reagan criteria (Fig. 2 and Online Resource Table 4). A $\beta 42 / A \beta 40$ was decreased in association with severe neuritic plaque burden, but not across Braak stages. P-tau181 and P-tau231 were both increased in relation to neuritic plaque burden and Braak stage; however, P-tau181 increased across all three neuritic plaque grades, whereas P-tau231 increased sharply from no or sparse to mild neuritic plaques. This suggests that in plasma, P-tau231 may be altered earlier than P-tau181 in relation to neuritic plaque evolution. Both plasma P-tau biomarkers increased across Braak stages. Total tau was minimally increased in late-stage AD (Braak VI). NfL levels ranged widely and did not show a clear relationship to stages of AD pathology determined by these approaches.

Within a subset of 76 participants who had a researchgrade MRI within 24 months of an available plasma biomarker assessment, we compared ROC metrics for P-tau plasma biomarkers and for MRI measures of entorhinal thickness and hippocampal volume (adjusted for total intracranial volume). Results are shown in Fig. 3, Supplementary on-line resource. Plasma P-tau181 and 231 had higher AUC values than the MRI indices for distinguishing between High ADNC pathology and Other Pathology. A model including both entorhinal thickness and P-tau 181 had only marginally better performance than P-tau181 alone. 
Table 1 Subject characteristics at final blood draw across neuropathology groups

\begin{tabular}{|c|c|c|c|c|c|c|c|}
\hline & Low pathology & $\begin{array}{l}\text { Intermediate } \\
\text { ADNC }\end{array}$ & High ADNC & Other pathology & $\begin{array}{l}\text { Intermediate } \\
\mathrm{ADNC}+\text { other }\end{array}$ & $\begin{array}{l}\text { High } \\
\text { ADNC+ other }\end{array}$ & $\begin{array}{l}\text { ANOVA/Chi-Sq } \\
p \text { value }\end{array}$ \\
\hline$n$ & 29 & 19 & 124 & 45 & 29 & 66 & \\
\hline Age at baseline & $83.6 \pm 6.9$ & $85.3 \pm 7.1$ & $74.8 \pm 9.4$ & $77.8 \pm 8.3$ & $79.1 \pm 6.3$ & $77.1 \pm 7.2$ & $1.5 \times 10^{-8} \mathrm{~b}, \mathrm{c}, \mathrm{e}, \mathrm{f}, \mathrm{g}, \mathrm{i}$ \\
\hline $\begin{array}{l}\text { Age at last } \\
\text { plasma }\end{array}$ & $86.8 \pm 6.1$ & $89 \pm 5.9$ & $77.3 \pm 9.9$ & $80.1 \pm 8.9$ & $81.3 \pm 6.7$ & $79.4 \pm 7.6$ & $2.4 \times 10^{-9} \mathrm{~b}, \mathbf{c}, \mathbf{e}, \mathbf{f}, \mathbf{g}, \mathbf{h}, \mathbf{i}$ \\
\hline Age at death & $88.7 \pm 6.2$ & $91 \pm 6.4$ & $79.8 \pm 9.6$ & $81.9 \pm 9.2$ & $82.8 \pm 6.9$ & $81.8 \pm 7.8$ & $2.0 \times 10^{-8}$ b,c,e,f,g,h,i \\
\hline $\begin{array}{l}\text { Last blood } \\
\text { draw to death } \\
\text { (years) }\end{array}$ & $1.9 \pm 1.2$ & $2 \pm 1.3$ & $2.5 \pm 1.4$ & $1.9 \pm 1.2$ & $1.5 \pm 1.1$ & $2.4 \pm 1.3$ & $7.5 \times 10^{-4 \mathrm{k}, \mathrm{o}}$ \\
\hline Female & $14(48 \%)$ & $8(42 \%)$ & $47(38 \%)$ & $8(18 \%)$ & $11(38 \%)$ & $18(27 \%)$ & 0.06 \\
\hline Hispanic & $5(17 \%)$ & $3(16 \%)$ & $10(8 \%)$ & $3(7 \%)$ & $3(10 \%)$ & $3(5 \%)$ & 0.39 \\
\hline $\begin{array}{l}\text { Education } \\
\text { (years) }\end{array}$ & $14.8 \pm 3.2$ & $15.4 \pm 5$ & $15.2 \pm 3.4$ & $15.5 \pm 3.3$ & $15.6 \pm 3.4$ & $15.8 \pm 2.7$ & 0.83 \\
\hline $\begin{array}{l}\text { APOE } 0 \text { e } 4 \\
\text { alleles }\end{array}$ & $20(69 \%)$ & $9(47 \%)$ & $51(41 \%)$ & $29(64 \%)$ & $14(48 \%)$ & $26(39 \%)$ & $0.021^{b, e, o}$ \\
\hline $\begin{array}{l}\text { APOE } 1 \text { e } 4 \\
\text { allele }\end{array}$ & $9(31 \%)$ & $10(53 \%)$ & $57(46 \%)$ & $14(31 \%)$ & $14(48 \%)$ & $31(47 \%)$ & \\
\hline $\begin{array}{l}\text { APOE } 2 \text { e } 4 \\
\text { alleles }\end{array}$ & $0(0 \%)$ & $0(0 \%)$ & $16(13 \%)$ & $2(4 \%)$ & $1(3 \%)$ & $9(14 \%)$ & \\
\hline Baseline MMSE & $27.9 \pm 2.1$ & $25.1 \pm 4.6$ & $21.9 \pm 6.1$ & $24.1 \pm 4.9$ & $22.4 \pm 5.8$ & $21.9 \pm 5.3$ & $1.7 \times 10^{-6 b, c, d, e}$ \\
\hline Baseline DRS & $131.2 \pm 10.3$ & $121.2 \pm 14.9$ & $109.8 \pm 23.7$ & $116.9 \pm 17.6$ & $112.7 \pm 18.9$ & $112.9 \pm 16.3$ & $1.8 \times 10^{-5 b, c, d, e}$ \\
\hline $\begin{array}{l}\text { Baseline CDR- } \\
\text { sb }\end{array}$ & $3.7 \pm 4.1$ & $5.3 \pm 4.5$ & $6.3 \pm 3.8$ & $5.9 \pm 4$ & $5.6 \pm 4.3$ & $6.3 \pm 3.6$ & 0.20 \\
\hline Last MMSE & $27.1 \pm 3.4$ & $24.2 \pm 4.1$ & $15.1 \pm 7.7$ & $22.4 \pm 5.4$ & $18.1 \pm 6.7$ & $16.8 \pm 5.7$ & $1.1 \times 10^{-20} b, c, d, \mathrm{~d}, \mathrm{e}, \mathrm{f}, \mathrm{h}, \mathrm{i}, \mathrm{j}, \mathrm{n}$ \\
\hline Last DRS & $128 \pm 13$ & $121 \pm 13.7$ & $86.1 \pm 31.9$ & $105.7 \pm 27.6$ & $96.6 \pm 30.9$ & $89.1 \pm 23.9$ & $1.1 \times 10^{-13} \mathbf{b , c}, \mathbf{d}, \mathbf{e}, \mathbf{f}, \mathbf{h}, \mathbf{I}, \mathbf{j}, \mathbf{n}$ \\
\hline Last CDR-sb & $2.8 \pm 4.3$ & $5.9 \pm 5.1$ & $10.9 \pm 4.4$ & $7.4 \pm 5.1$ & $8.2 \pm 4.8$ & $10.3 \pm 4.5$ & $7.6 \times 10^{-15}$ b,c,d,e,f,I, , j,k,k,n \\
\hline $\begin{array}{l}\text { Last clinical } \\
\text { diagnosis: } \\
\text { normal }\end{array}$ & $18(62 \%)$ & $3(16 \%)$ & $1(1 \%)$ & $5(11 \%)$ & $0(0 \%)$ & $0(0 \%)$ & 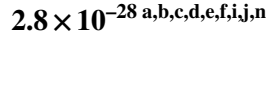 \\
\hline MCI & $4(14 \%)$ & $4(21 \%)$ & $1(1 \%)$ & $2(4 \%)$ & $2(7 \%)$ & $2(3 \%)$ & $7.6 \times 10^{-4 b, f}$ \\
\hline $\mathrm{AD} /$ dementia & $7(24 \%)$ & $12(63 \%)$ & $108(87 \%)$ & $16(36 \%)$ & $16(55 \%)$ & $51(77 \%)$ & $3.4 \times 10^{-14} \mathrm{a}, \mathrm{b}, \mathrm{d}, \mathrm{e}, \mathbf{f}, \mathbf{j}, \mathbf{k}, \mathbf{n}, \mathrm{o}$ \\
\hline DLB/PDD & $0(0 \%)$ & $0(0 \%)$ & $8(6 \%)$ & $21(47 \%)$ & $10(34 \%)$ & $11(17 \%)$ & $6.8 \times 10^{-11} \mathrm{c}, \mathrm{d}, \mathrm{g}, \mathrm{h}, \mathbf{j}, \mathrm{k}, \mathrm{n}$ \\
\hline FTLD & $0(0 \%)$ & $0(0 \%)$ & $5(4 \%)$ & $1(2 \%)$ & $1(3 \%)$ & $2(3 \%)$ & 0.84 \\
\hline Other** & $0(0 \%)$ & $0(0 \%)$ & $1(1 \%)$ & $0(0 \%)$ & $0(0 \%)$ & $0(0 \%)$ & 0.91 \\
\hline \# of blood draws & $2.6 \pm 1.6$ & $2.5 \pm 1.8$ & $2.5 \pm 1.3$ & $2.4 \pm 1.4$ & $2.4 \pm 1.6$ & $2.3 \pm 1.1$ & 0.98 \\
\hline \# of annual visits & $6.3 \pm 3.1$ & $5.2 \pm 2.7$ & $4.5 \pm 2.3$ & $4.3 \pm 2.6$ & $4 \pm 2.4$ & $3.9 \pm 2.3$ & $3.0 \times 10^{-4} \mathrm{~b}, \mathrm{c}, \mathrm{d}, \mathrm{e}$ \\
\hline
\end{tabular}

Tukey HSD post hoc comparisons significant with adjusted $p<0.05$, or pairwise Chi-square tests with Benjamin-Hochberg adjusted $p<0.05$ Missing data: Hispanic $(n=1,<1 \%)$, first MMSE $(n=5,1 \%)$, first DRS $(n=8, n \%)$, first CDR-sb $(n=31,10 \%)$, last MMSE $(n=8,2 \%)$, last DRS $(n=12,4 \%)$, last CDR-sb $(n=12,4 \%)$

MMSE Mini-Mental State Exam, DRS Dementia Rating Scale, CSR-sb Clinical Dementia Rating-sum of boxes

**Other clinical diagnosis was vascular dementia $(n=1)$

${ }^{a}$ Low Path vs Intermediate ADNC, ${ }^{b}$ Low Path vs High ADNC, ${ }^{c}$ Low Path vs Other Path, ${ }^{\mathrm{d}}$ Low Path vs Intermediate ADNC + Other, ${ }^{\mathrm{e}}$ Low Path vs High ADNC+ Other, ${ }^{f}$ Intermediate ADNC vs High ADNC, ${ }^{g}$ Intermediate ADNC vs Other Path, hIntermediate ADNC vs Intermediate $\mathrm{ADNC}+$ Other, ${ }^{\mathrm{i}}$ Intermediate ADNC vs High ADNC + Other, ${ }^{\mathrm{j}} \mathrm{High}$ ADNC vs Other Path, ${ }^{\mathrm{k}} \mathrm{High}$ ADNC vs Intermediate ADNC + Other, ${ }^{\mathrm{l}} \mathrm{High}$ ADNC vs High ADNC + Other, ${ }^{\mathrm{m}}$ Other Path vs Intermediate ADNC+ Other, ${ }^{\mathrm{n}}$ Other Path vs High ADNC+ Other

${ }^{\circ}$ Intermediate $\mathrm{ADNC}+$ Other vs High $\mathrm{ADNC}+$ Other

We examined plasma biomarkers in other common age-related pathologies that can cause dementia, including two key pathologic features of LATE: HS which has been assessed on all cases) and abnormal hippocampal TDP-43 accumulation of LATE (which was assessed in a subset-see below), and LBD (defined as limbic or neocortical stages of $\alpha$-synuclein pathology). We made the following comparisons: (1) HS without High ADNC, HS with High ADNC, and High ADNC without HS; LATENC without High ADNC, LATE-NC with High ADNC, 
Table 2 Neuropathology: staging and subtypes

Low Pathology Intermediate ADNC High ADNC Other Path Intermediate

High ADNC+ Other $\mathrm{ADNC}+$ Other

\begin{tabular}{|c|c|c|c|c|c|c|}
\hline$n$ & 29 & 19 & 124 & 45 & 29 & 66 \\
\hline Neuritic plaques: sparse & $13(45 \%)$ & $0(0 \%)$ & $0(0 \%)$ & $31(69 \%)$ & $0(0 \%)$ & $0(0 \%)$ \\
\hline Neuritic plaques: moderate & $16(55 \%)$ & $16(84 \%)$ & $32(26 \%)$ & $12(27 \%)$ & $20(69 \%)$ & $21(32 \%)$ \\
\hline Neuritic plaques: frequent & $0(0 \%)$ & $3(16 \%)$ & $92(74 \%)$ & $2(4 \%)$ & $9(31 \%)$ & $45(68 \%)$ \\
\hline Braak 0-II & $27(93 \%)$ & $0(0 \%)$ & $0(0 \%)$ & $39(87 \%)$ & $0(0 \%)$ & $0(0 \%)$ \\
\hline Braak III-IV & $2(7 \%)$ & $19(100 \%)$ & $0(0 \%)$ & $6(13 \%)$ & $29(100 \%)$ & $0(0 \%)$ \\
\hline Braak V-VI & $0(0 \%)$ & $0(0 \%)$ & $124(100 \%)$ & $0(0 \%)$ & $0(0 \%)$ & $66(100 \%)$ \\
\hline NIA-Reagan: not/Low ADNC & $29(100 \%)$ & $0(0 \%)$ & $0(0 \%)$ & $45(100 \%)$ & $0(0 \%)$ & $0(0 \%)$ \\
\hline NIA-Reagan: Intermediate ADNC & $0(0 \%)$ & $19(100 \%)$ & $0(0 \%)$ & $0(0 \%)$ & $29(100 \%)$ & $0(0 \%)$ \\
\hline NIA-Reagan: High ADNC & $0(0 \%)$ & $0(0 \%)$ & $124(100 \%)$ & $0(0 \%)$ & $0(0 \%)$ & $66(100 \%)$ \\
\hline Thal phase $0-2$ & $2(7 \%)$ & $1(5 \%)$ & $0(0 \%)$ & $4(9 \%)$ & $0(0 \%)$ & $1(2 \%)$ \\
\hline Thal phase 3 & $0(0 \%)$ & $1(5 \%)$ & $0(0 \%)$ & $1(2 \%)$ & $0(0 \%)$ & $1(2 \%)$ \\
\hline Thal phase $4-5$ & $1(3 \%)$ & $3(16 \%)$ & $50(40 \%)$ & $4(9 \%)$ & $10(34 \%)$ & $30(45 \%)$ \\
\hline CAA: none/mild & $20(69 \%)$ & $7(37 \%)$ & $46(37 \%)$ & $34(76 \%)$ & $15(52 \%)$ & $22(33 \%)$ \\
\hline CAA: moderate & $5(17 \%)$ & $8(42 \%)$ & $37(30 \%)$ & $8(18 \%)$ & $7(24 \%)$ & $23(35 \%)$ \\
\hline CAA: severe & $4(14 \%)$ & $4(21 \%)$ & $41(33 \%)$ & $3(7 \%)$ & $7(24 \%)$ & $21(32 \%)$ \\
\hline Diffuse plaques: sparse & $12(41 \%)$ & $0(0 \%)$ & $10(8 \%)$ & $26(58 \%)$ & $0(0 \%)$ & $2(3 \%)$ \\
\hline Diffuse plaques: moderate & $12(41 \%)$ & $7(37 \%)$ & $19(15 \%)$ & $6(13 \%)$ & $10(34 \%)$ & $11(17 \%)$ \\
\hline Diffuse plaques: frequent & $5(17 \%)$ & $12(63 \%)$ & $95(77 \%)$ & $13(29 \%)$ & $19(66 \%)$ & $53(80 \%)$ \\
\hline LBD: brainstem & $0(0 \%)$ & $0(0 \%)$ & $0(0 \%)$ & $6(13 \%)$ & $3(10 \%)$ & $4(6 \%)$ \\
\hline LBD: limbic & $0(0 \%)$ & $0(0 \%)$ & $0(0 \%)$ & $9(20 \%)$ & $1(3 \%)$ & $7(11 \%)$ \\
\hline LBD: neocortical & $0(0 \%)$ & $0(0 \%)$ & $0(0 \%)$ & $17(38 \%)$ & $17(59 \%)$ & $31(47 \%)$ \\
\hline Hippocampal sclerosis & $0(0 \%)$ & $0(0 \%)$ & $0(0 \%)$ & $14(31 \%)$ & $8(28 \%)$ & $28(42 \%)$ \\
\hline FTLD & $0(0 \%)$ & $0(0 \%)$ & $0(0 \%)$ & $4(9 \%)$ & $1(3 \%)$ & $4(6 \%)$ \\
\hline Other Pathology** & $0(0 \%)$ & $0(0 \%)$ & $0(0 \%)$ & $4(9 \%)$ & $0(0 \%)$ & $3(5 \%)$ \\
\hline Vascular including microinfarcts & $11(38 \%)$ & $8(42 \%)$ & $24(19 \%)$ & $6(13 \%)$ & $4(14 \%)$ & $5(8 \%)$ \\
\hline
\end{tabular}

Missing data: Thal phase $(n=196,63 \%)$

**Other Pathology: neurodegeneration with brain iron accumulation $(n=3)$, multiple sclerosis $(n=1)$, limbic microglial nodular encephalitis $(n=1)$, atypical tauopathy with degeneration of substantia nigra $(n=1)$, alcoholic brain degeneration $(n=1)$

and High ADNC without LATE-NC; and (3) LBD without High ADNC, LBD with High ADNC, and High ADNC without LBD. In HS, P-tau181 and P-tau231 were increased when High ADNC was present while NfL was slightly increased in HS with or without ADNC compared to ADNC alone (Fig. 3a, Online Resource Table 5). We fully staged LATE-NC in 64 cases and performed hippocampal TDP-43 immunohistochemistry in 52 additional cases selected to oversample HS and Intermediate ADNC. Summary data on this set of 116 cases are shown in Online Resource Table 6, and comparisons with plasma biomarkers in Fig. 3b. Relationships between higher levels of P-tau biomarkers in plasma and ADNC remained, whereas there were no significant associations between any of the plasma biomarkers and LATE-NC in the hippocampus. The main findings for LBD were that plasma A $\beta 42 / 40$ was slightly decreased, and P-tau181 and P-tau231 significantly increased, if High ADNC was also present (Fig. 3c).
We performed ROC analyses to determine classification accuracy of the various plasma biomarkers using pathologically defined Low vs High ADNC groups as the "gold standard" to define cutoffs. Because of the older age and stability of plasma biomarkers from baseline to last blood draw in the low AD pathology group, we compared baseline plasma biomarkers in this group to last-blood-draw plasma biomarkers in the high likelihood AD group to achieve closer age matching. As shown in Fig. 4, the plasma biomarkers with the best classification accuracy were plasma P-tau181 followed by P-tau231. P-tau231 had relatively lower sensitivity than $\mathrm{P}$-tau181 in these comparisons because it was increased in more people who died with Braak stage 0 , I and II pathology than was P-tau181 (similar to relationships found in prior imaging studies where P-tau231 was increased as early as Braak stage II defined by Tau PET) [3].

We modeled longitudinal changes in the various plasma biomarkers from baseline to death in relation to severity of 

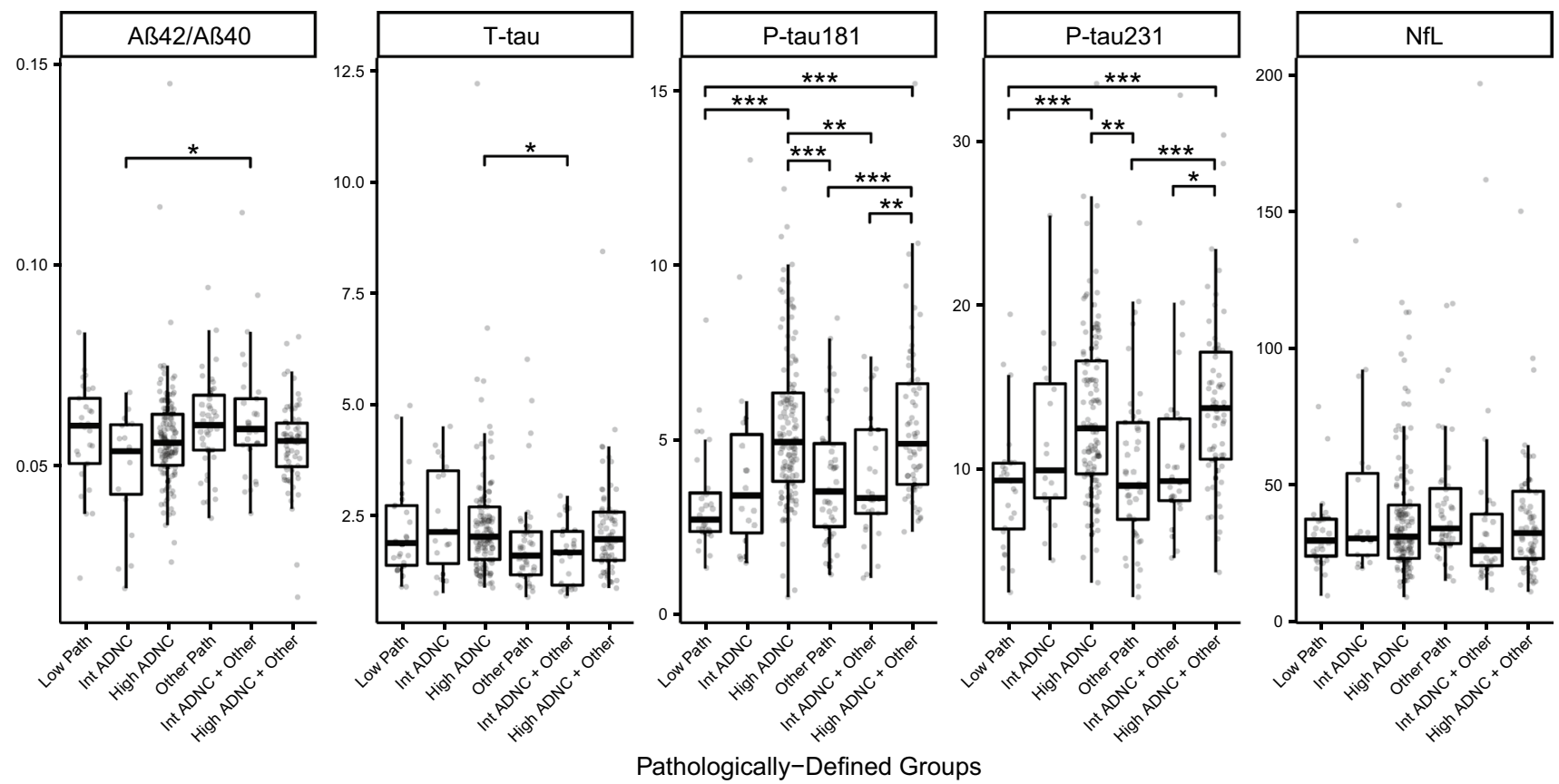

Fig. 1 Plasma biomarkers at last blood draw by pathologic groups. Boxplots of the distributions of the plasma biomarkers from the blood draw closest to death by pathologic group. One NfL value of 1154 in FTLD patient was removed from plots for visualization but retained in statistical analyses. Effect sizes and both raw and multiple-com- parisons adjusted $p$ values are available in Supplementary Table 2, On-line Resource. Statistics for pairwise comparisons are corrected for multiple comparisons using Tukey's method to maintain a family error rate of 0.05 , and are graphically summarized as follows: $* p<0.05, * * p<0.01, * * * p<0.001$

in all subjects with AD pathology (Fig. 6b-d), we found that cognitive decline was steeper in patients with high (i.e., above our ROC-defined cutoffs) baseline plasma P-tau181 $(>3.64 \mathrm{pg} / \mathrm{mL})$ or P-tau231 (> $10.5 \mathrm{pg} / \mathrm{mL})$ levels than in those with low (below cutoff) levels of these plasma biomarkers. Although NfL had low sensitivity as a diagnostic biomarker, levels above the diagnostic cutoff strongly predicted faster cognitive decline. Analyses of baseline plasma $A \beta 42 / 40$ did not strongly predict cognitive decline (data not shown). Similar results were found for analyses of predictors of progression on other clinical indices, e.g., CDR-sb (data not shown). In exploratory analyses of cognitive change in people who were assessed as having normal cognition or MCI at baseline, higher plasma P-tau181, 231 and NfL were associated with more rapid cognitive decline (Fig. 7a-d). Higher baseline P-tau231 levels showed weaker statistical significance, but levels at baseline were associated with lower DRS scores at baseline. Since NfL did not have strong associations with AD, LBD, HS, or LATE pathological changes, we assessed whether there were differences in vascular risk factors or other characteristics of the high vs low NfL subgroups. Those with high plasma NfL were more likely to have atrial fibrillation and history of TIA or stroke and were more likely to have microinfarcts and moderate-tosevere atherosclerosis of the circle of Willis (Table 3). These suggest that an additional burden of vascular risk and brain 

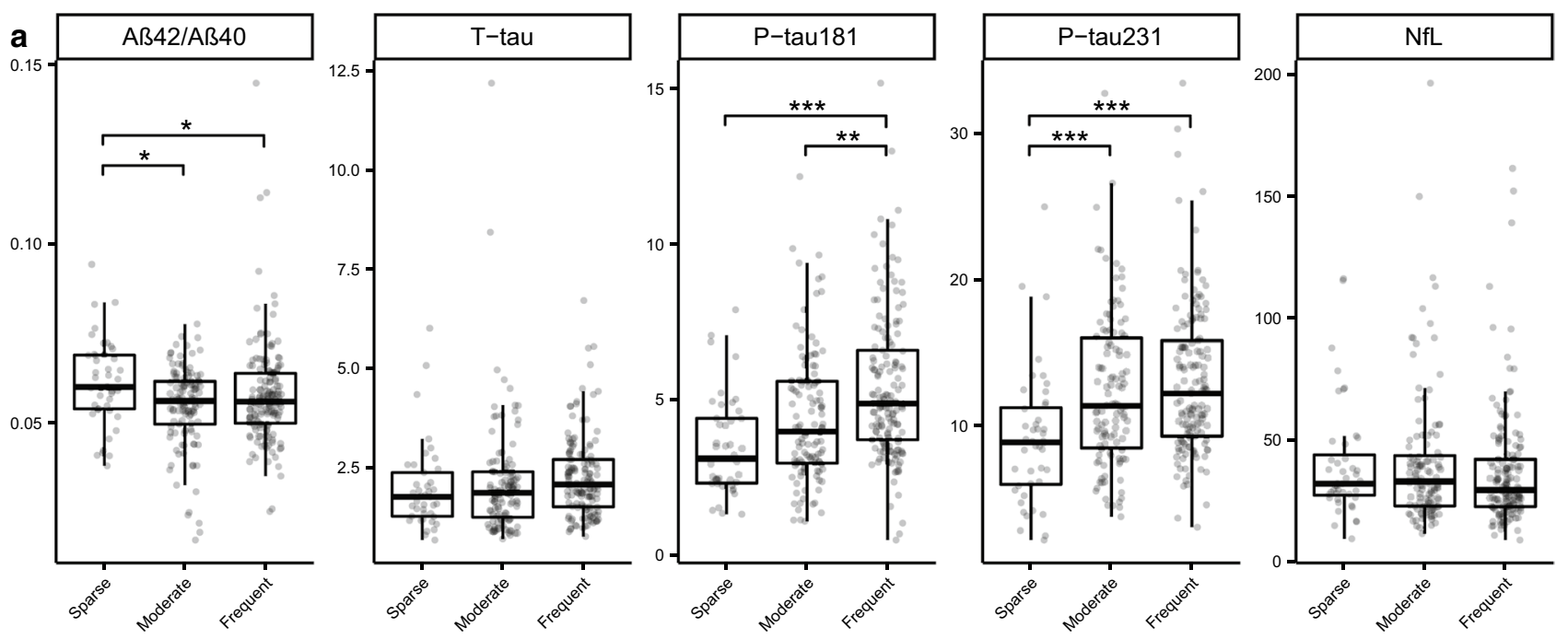

CERAD Neuritic Plaque Density at Autopsy
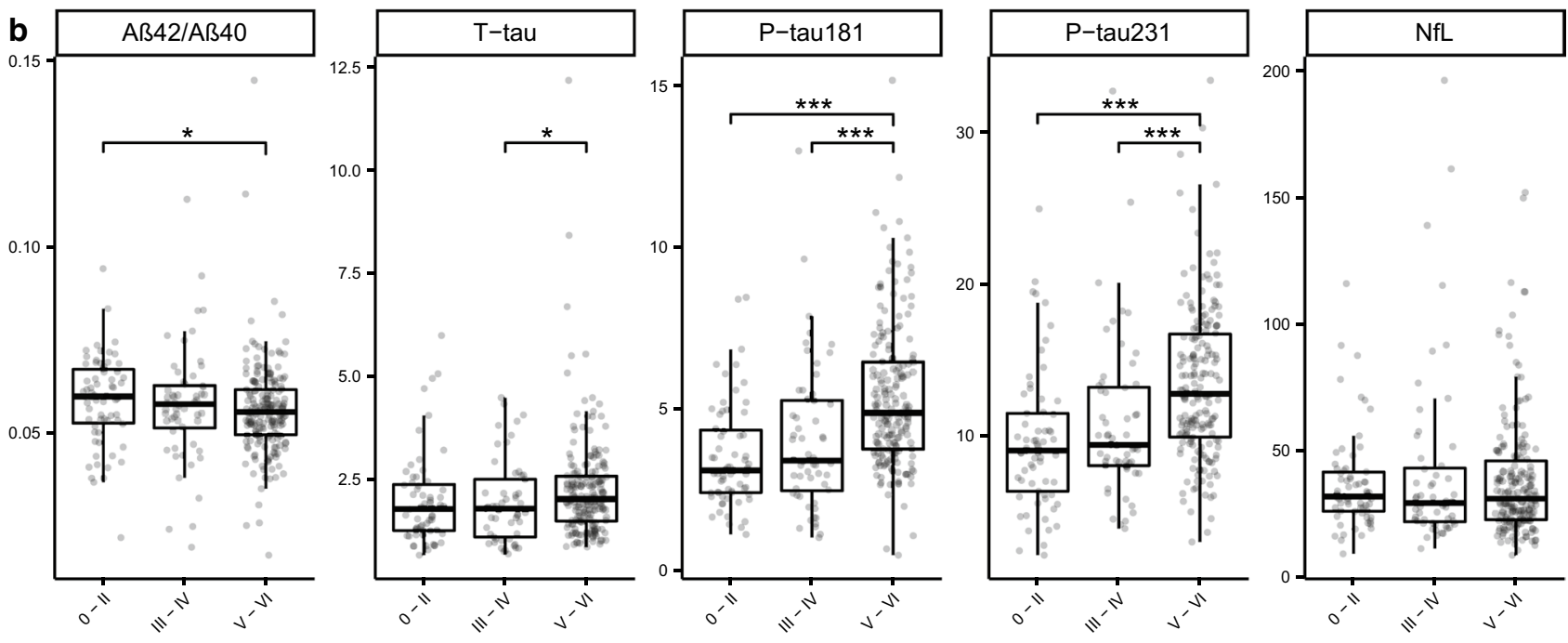

Braak Stage at Autopsy
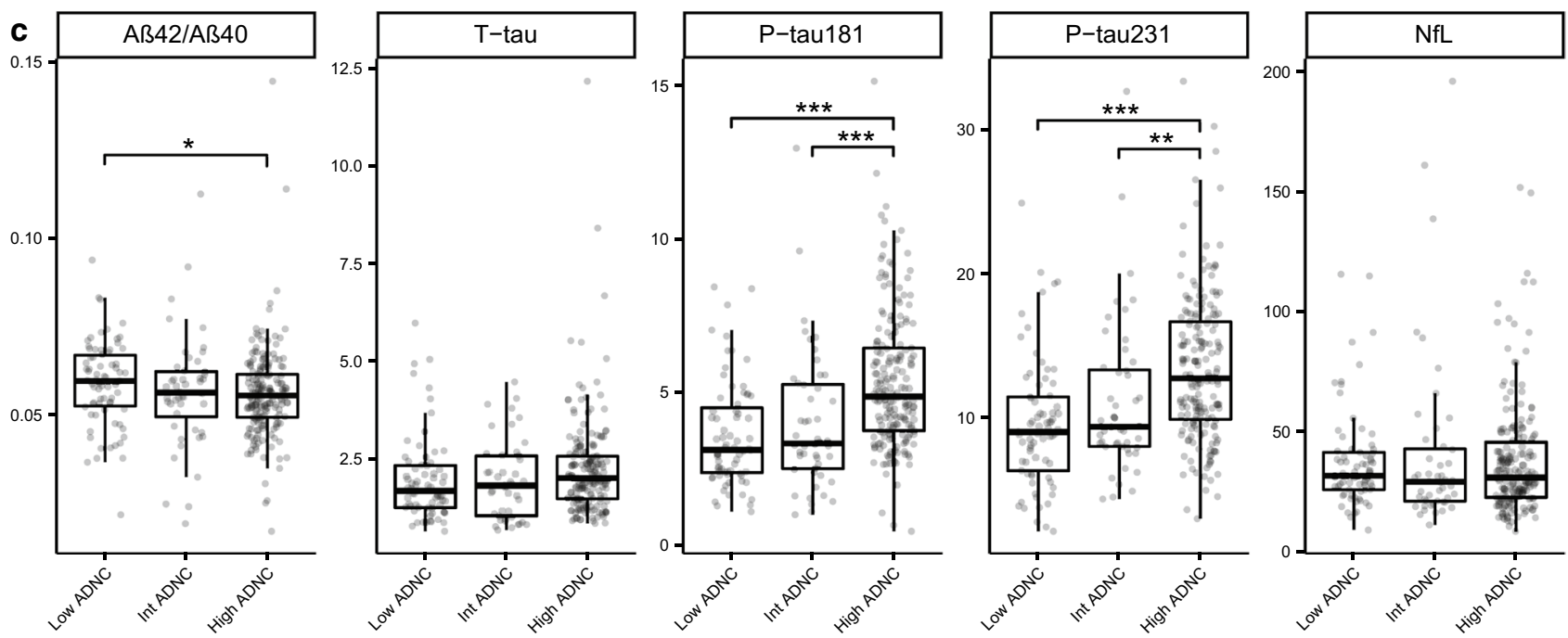

NIA-Reagan Criteria for AD 
4Fig. 2 Plasma biomarkers in groups defined by different staging of AD Neuropathology. Boxplots of the distributions of the plasma biomarkers from the blood draw closest to death divided by a CERAD neuritic plaque density score, b Braak neurofibrillary tangle stage, and c NIA-Reagan Institute criteria stage of ADNC. One NfL value of 1154 in FTLD patient was removed from plots for visualization, but retained in statistical analyses. Effect sizes and both raw and multiple-comparisons adjusted $p$ values are available in Supplementary Table 3, On-line Resource. Statistics for pairwise comparisons are corrected for multiple comparisons using Tukey's method to maintain a family error rate of 0.05 , and are graphically summarized as follows: ${ }^{*} p<0.05,{ }^{* *} p<0.01,{ }^{* * *} p<0.001$

pathology may contribute to the increased levels of $\mathrm{NfL}$ in plasma associated with neurodegeneration.

When we modeled trajectories of changes in plasma biomarkers across the spectrum of AD pathology (Fig. 8), we found that both P-tau231 and P-tau181 showed early increases, with P-tau231 changing slightly earlier relative to P-tau181. There was an increase in plasma levels of P-tau231 in some subjects without dementia who had low or intermediate tau pathology, suggesting that these early stages of $\mathrm{AD}$ regional pathology may be sufficient to cause the release of enough P-tau231 to be detectable in plasma. Total tau did not show a consistent pattern of longitudinal changes in relation to different grades of tau pathology.

\section{Discussion}

Plasma ATN biomarkers show great promise for AD diagnosis and staging. Many studies have correlated plasma biomarkers with amyloid and tau PET imaging, CSF biomarkers, and cognitive staging. Relatively few, however, have analyzed plasma in relation to autopsy brain findings. Our study is the largest autopsy series to date, which allowed us to validate longitudinal plasma biomarkers against autopsyconfirmed diagnoses, examine specific associations with $\mathrm{AD}$ and other pathologies, and compare performance of plasma total tau, P-tau181 and P-tau231.

The influences of age and sex on plasma biomarkers have been analyzed in various cohorts. It may be difficult to distinguish aging effects from those of preclinical pathology (e.g., A $\beta 42$ ). However, we found a significant effect of age on plasma $A \beta 42$, a weak effect of age on plasma NfL, and weak effect of age on plasma P-tau181 and P-tau231 (age was included in our models). We found no significant effect of sex on any of the plasma biomarkers.

Several studies using immunoprecipitation-mass spectrometry (IP-MS) have shown that the ratio of plasma levels of $A \beta x-42 / x-40$ or $A \beta 1-42 / 1-40[19,28,35,40]$ is related to cerebral amyloidosis measured by PET, with AUCs from ROC analyses of approximately $0.7-0.8$; this improved to 0.8 or higher in studies where age and $A P O E$ genotype were included in the algorithm. We obtained a lower AUC of 0.60 for plasma A $\beta 1-42 / 1-40$ measured by a Simoa assay. This was similar to findings from another recent pathology series [7] and comparable to the Simoa results, but lower than IP-MS assay results, in a recent study that compared Simoa and IP-MS [18]. In the prior autopsy study and our study, Thal phase evaluation of amyloid pathology was available on a minority of subjects. Amyloid accumulation in diffuse plaques and cerebrovascular deposits occurs commonly in aging and this source contributes to the overall amyloid burden detectable by amyloid PET [14] and to Thal phase score [38]. Thus, it is not clear that Thal phase allows a more sensitive look into the relationship between amyloid and cognition or dementia than indices such as CERAD neuritic plaque density [41]. In CSF, levels of $A \beta 42$ and A $\beta 42 / 40$ appear to decrease before amyloid PET reaches significant thresholds [11, 23, 32], but it is challenging to determine thresholds and identify cutoff points for plasma A $\beta 42 / 40$ which produces a much smaller effect size than CSF A $\beta 42 / 40$ (difference between amyloid positive vs negative in relation to the overall distribution of values) [40]. This may be even more difficult among older people, many of whom harbor intermediate but subthreshold amyloid pathology. We found that plasma $A \beta 42 / 40$ was lower in cases with significant amyloid pathology (moderate to severe by CERAD or in the limited set of patients, vs Thal phase), but with much overlap.

P-tau181 and P-tau231 performed comparably well in pathological comparisons of groups with or without $\mathrm{AD}$ neurofibrillary pathology, whereas total tau showed a small group increase only in people with severe (Braak stage VI) tau pathology. A previous study noted that P-tau231, measured using the same Simoa assay as in this study, showed high diagnostic value based on Tau PET, CSF amyloid and tau classification, and autopsy [3]. P-tau231 also showed increases earlier than P-tau181 and was able to discriminate Braak 0 vs Braak I-II stages. We had relatively few Braak 0 stage patients to allow this comparison, but still found that P-tau231 showed an earlier increase during intermediate stages of neuritic plaque pathology than P-tau181. Levels of P-tau231 and P-tau181 were correlated in our series, with the strength of correlation similar in $\mathrm{AD}(r=0.69)$ and all cases $(r=0.68)$.

The timing of trajectories of changes in fragments of tau with different phospho-epitopes is of importance with regard to the potential to identify or track earlier stages in the development of AD. While levels of P-tau181, P-tau231 and P-tau217 are correlated in CSF [37], studies suggest that CSF P-tau217 and P-tau231 may show pathological changes earlier than P-tau181, and that CSF P-tau 217 correlates more strongly with amyloid and tau PET positivity than other forms of P-tau.[21]. Prior studies have identified relationships between plasma P-tau181 and amyloid PET 

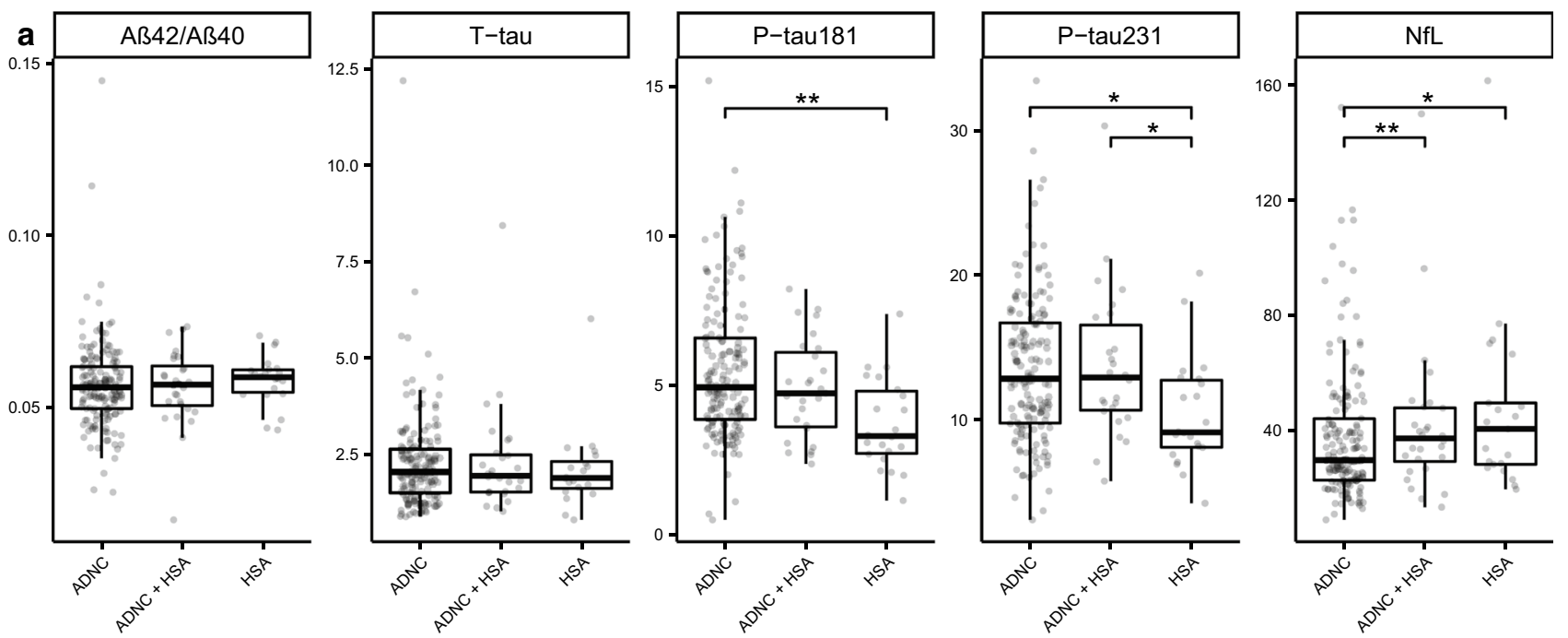

Pathology at Autopsy
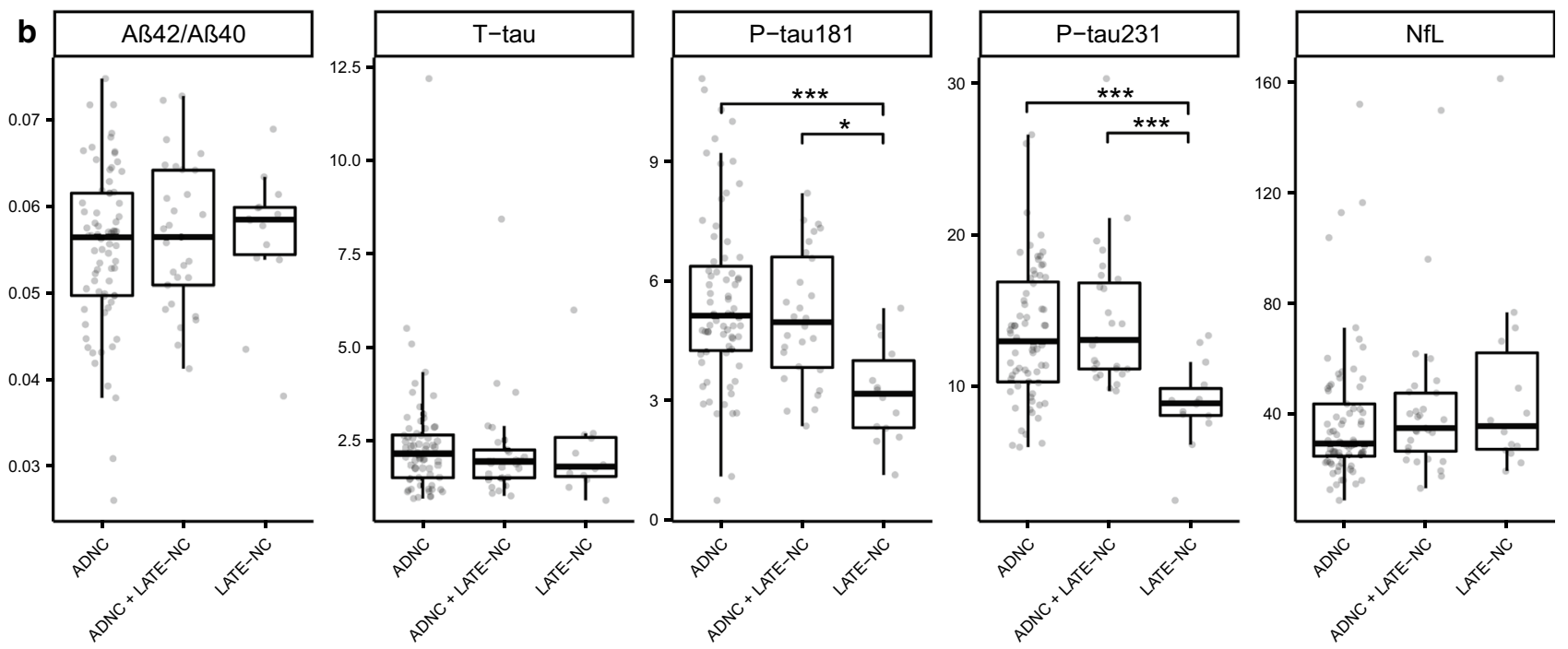

Pathology at Autopsy
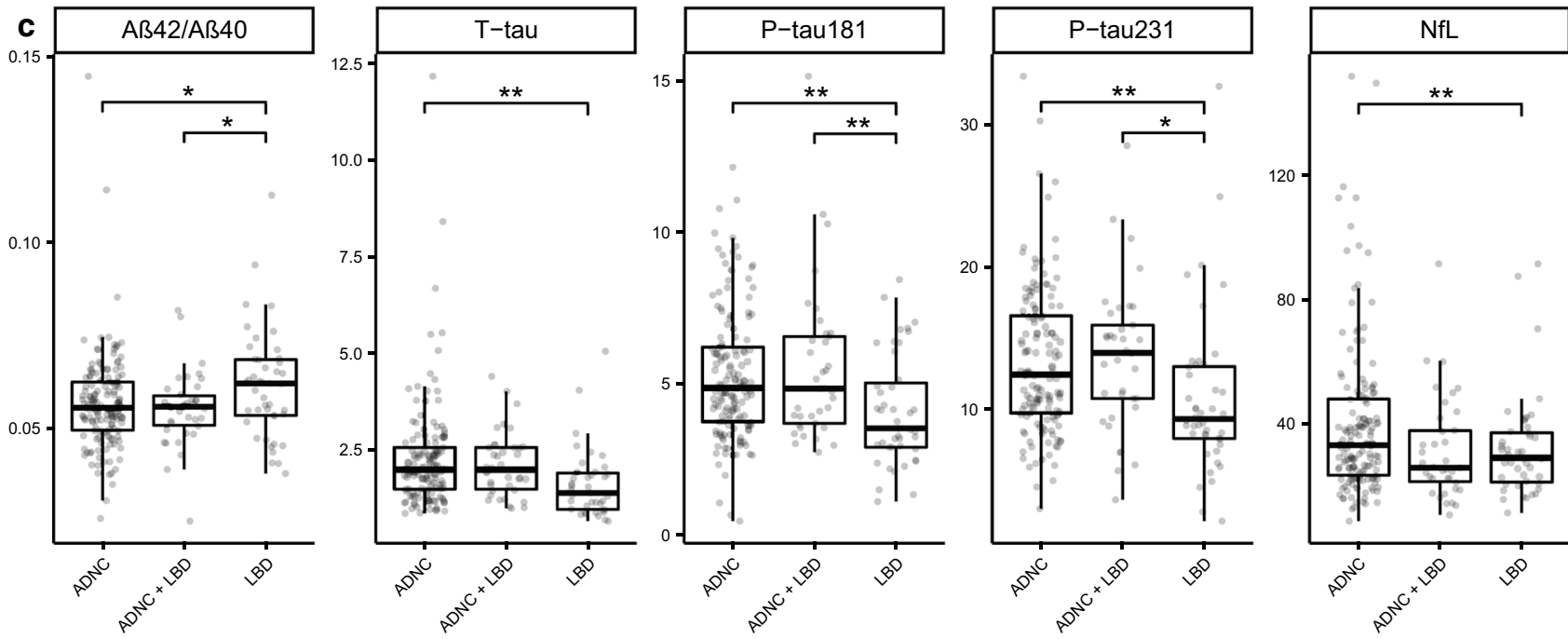

Pathology at Autopsy 
4Fig. 3 Plasma biomarkers in relation to Hippocampal sclerosis (HS), Limbic Age-related TDP-43 Encephalopathy (LATE), Lewy Body Disease (LBD) and ADNC. Boxplots of the distributions of the plasma biomarkers from the blood draw closest to death in individuals with High ADNC and/or other non-AD pathologies: a hippocampal sclerosis of aging defined as neuronal loss in the CA1 and subiculum out of proportion with the degree of AD pathology, b hippocampal staining positive for TDP-43 proteinopathy representing LATE neuropathologic changes (LATE-NC), and c Lewy body disease of the limbic (transitional) or neocortical (diffuse) type. These plots and analyses exclude participants who did not have either High ADNC or the non-AD pathology being assessed. TDP-43 immunostaining was available in a select subset of cases, with their demographic data available in Supplementary Table 4, On-line Resource. One NfL value of 1154 in a FTLD patient was removed from plots for visualization, but retained in statistical analyses. Effect sizes and both raw and multiple-comparisons adjusted $p$ values are available in Supplementary Table 5, On-line Resource. Statistics for pairwise comparisons are corrected for multiple comparisons using Tukey's method to maintain a family error rate of 0.05 , and are graphically summarized as follows: $* p<0.05, * * p<0.01, * * * p<0.001$

positivity, but these relationships were weaker in cognitively normal people than in those with MCI or dementia $[17,25$, 30]. P-tau231 was shown to be increased in plasma at lower levels of amyloid PET positivity than P-tau181, and in other studies increases in plasma P-tau217 appeared slightly earlier than in P-tau181 [25, 31]. In our study, longitudinal modeling of plasma P-tau231 and P-tau181 showed that both were significantly increased as early as 10 years before death in people who eventually showed clear AD pathology (i.e., NIA-Reagan High Likelihood) and continued to increase in the years leading up to death, and those levels were significantly higher than in people who died with low or intermediate AD pathology. These findings are similar to those recently reported for P-tau181 [20]; however, the baseline level of cognitive impairment in our cohort was milder (MMSE 22-23/30 in AD and 26/30 in people with low or no AD pathology) than in the previous study. We found that P-tau231 and P-tau181 had slightly different modeled trajectories vs CERAD neuritic plaque density, with P-tau231 showing a clearer continued increase compared to P-tau181 in people who eventually died with intermediate AD pathology. Previous studies showed that plasma P-tau231 showed increases as early as Braak II stage equivalent on tau PET scan [3]. Consistent with these findings, plasma P-tau231 was above threshold, to a greater extent than P-tau181, in some people in the Braak I-II subgroup in our autopsy series.

Even in analyses limited to individuals with normal cognition or MCI at baseline, higher baseline levels of plasma P-tau181 and P-tau231 (for analytical purposes, divided at the ROC cutoffs) predicted decline on tests of global cognition during 5 years of follow-up. This replicates clinical studies suggesting the potential use of plasma biomarkers, particularly forms of P-tau, as early predictive biomarkers of cognitive decline and dementia [8, 17, 33]. Although plasma NfL performed weakly relative to P-tau181 or 231 as a diagnostic biomarker, NfL levels above the cutoff predicted faster progression. Within our relatively small series, this suggests that there may be a subset of people with AD who have more intense neurodegeneration, reflected by NfL, which may, therefore, complement P-tau biomarkers as a predictor of cognitive decline. Further support for NfL as a predictor comes from data analyses from the BioFINDER study [8] and the Alzheimer's Disease Neuroimaging Initiative (ADNI), where higher levels of plasma NfL predicted faster cognitive decline over 5-6 years in people with subtle cognitive changes or MCI [4]. Our study also suggests that cerebrovascular disease mechanisms or pathology may contribute to higher levels of plasma NfL and more rapid cognitive decline.

Multiple brain pathologies are common in AD and may be associated with faster progression of dementia [5, 16]. We found that plasma amyloid and P-tau biomarkers showed similar patterns in people with DLB or LATE-NC: no changes when pure DLB or LATE-NC were present, but increases consistent with an $\mathrm{AD}$ profile when significant concomitant AD was present. Plasma NfL was not helpful in pointing to either of these additional pathologies. In a recent large study across multiple cohorts [1], plasma NfL was helpful in detecting MCI and AD (with small to medium effect sizes) and disorders such as FTD, progressive supranuclear palsy and corticobasal syndrome, and was slightly increased in DLB [1]. In our series, the Low Pathology group had a mean plasma NfL level of $32 \mathrm{pg} / \mathrm{mL}$, whereas in the multicenter study [1] an NfL level of $35 \mathrm{pg} / \mathrm{mL}$ was at the 90th percentile, and $38 \mathrm{pg} / \mathrm{mL}$ at the 95 th percentile, when amyloid-negative controls were used to derive cutoffs. Our ROC-derived plasma NfL cutoff was $36.5 \mathrm{pg} / \mathrm{mL}$, which has specificity of $90 \%$ (i.e., similar to the amyloidnegative control cutoffs in Ashton et al. [1]) and sensitivity of $42 \%$ for Braak stage VI AD. Plasma NfL sensitivity and specificity for this cutoff was higher for the small number of cases in our series with FTD pathology. NfL was slightly increased in association with HS pathology in the current study. Although this suggests that in an appropriate clinical setting a pattern of high plasma NfL and normal P-tau levels may point towards HS, there was too much overlap with ADNC to make this a useful discriminator.

Strengths of this study include the large number of subjects, standardization of clinical assessment, longitudinal plasma collection with harmonized preanalytical procedures and brain neuropathology assessment, and use of sensitive assays for plasma biomarkers. One limitation is the 


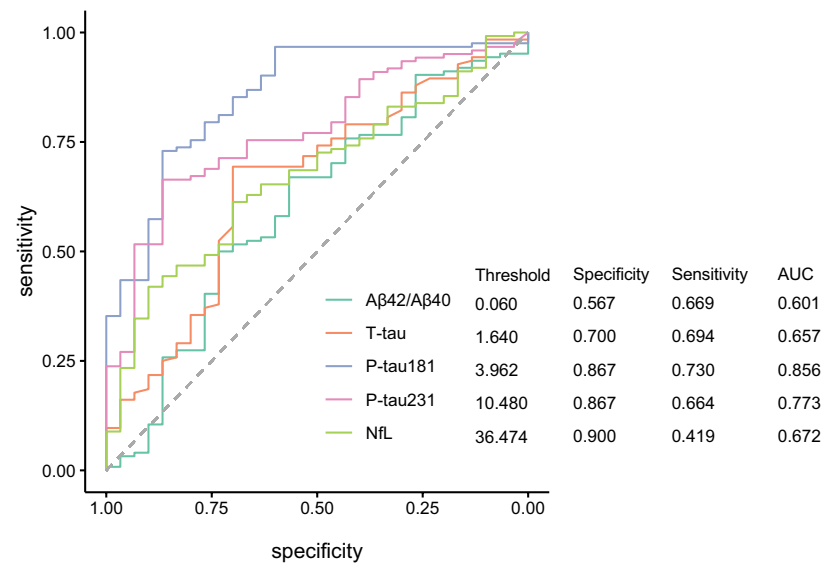

Fig. 4 ROC analyses, comparing plasma biomarkers in the Low Pathology group vs the last blood draw in the High ADNC group. ROC curves and associated thresholds, specificities, sensitivities, and areas under the curve (AUCs) for the use of each plasma biomarker to distinguish patients who were classified as Low Pathology at autopsy from those who were classified as High ADNC. Because of the older age and stability of plasma biomarkers from baseline to last blood draw in the Low Pathology group, baseline plasma biomarkers in this group were compared to the final blood draw High ADNC group to achieve closer age matching

interval from last blood draw to autopsy: although this was relatively short, there is a gap of a few years during which brain pathology may progress, complicating the analysis of biomarker-pathology correlations. In addition, it is difficult in a study of this nature to obtain subjects with normal cognition who are free of brain pathology, especially covering a wide age range, therefore, we had limited capability to directly determine when plasma biomarkers might change, and our model of inflection points is less precise than similar models from large-scale clinical studies. Clinical studies have advantages over autopsy studies when establishing cutoffs or examining effects of age on biomarkers, but for some questions they may be confounded by unknown co-pathologies.

This study provides support for plasma biomarkers in relation to diagnosis and prognosis based on the ATN framework. The biomarkers measured in our study do not identify other common pathologies that may occur on their own or together with AD pathology and may contribute to dementia, in particular a-synuclein and TDP-43 pathology. A potential biochemical marker for detecting DLB is $\alpha$-synuclein. RT-QuIC measures that detect $\alpha$-synuclein aggregates capable of seeding fibril formation have high accuracy when measured in CSF or skin biopsy samples but are not yet detectable in plasma [10, 24, 34]. Unfortunately, no specific biochemical marker for HS or LATE is available at present. The great progress that has been made in plasma biomarkers for ATN raises hope for developing a more extensive panel of plasma biomarkers to comprehensively profile other common brain pathologies in relation to cognitive decline. 


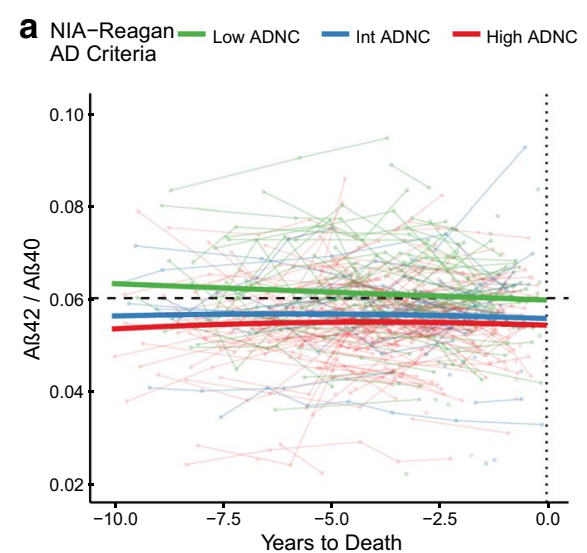
b NIA-Reagan - Low ADNC - Int ADNC - High ADNC
AD Criteria
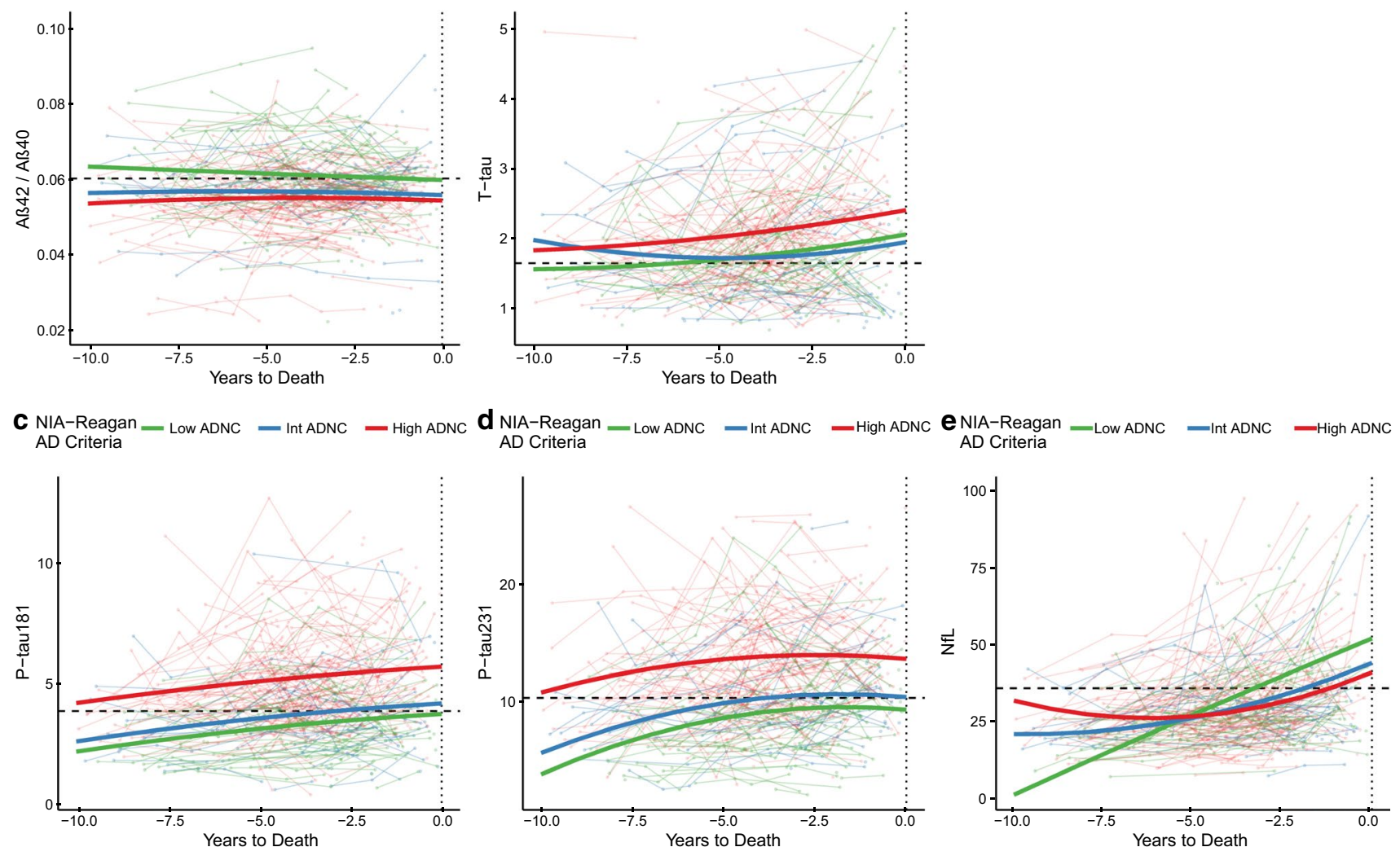
AD Criteria
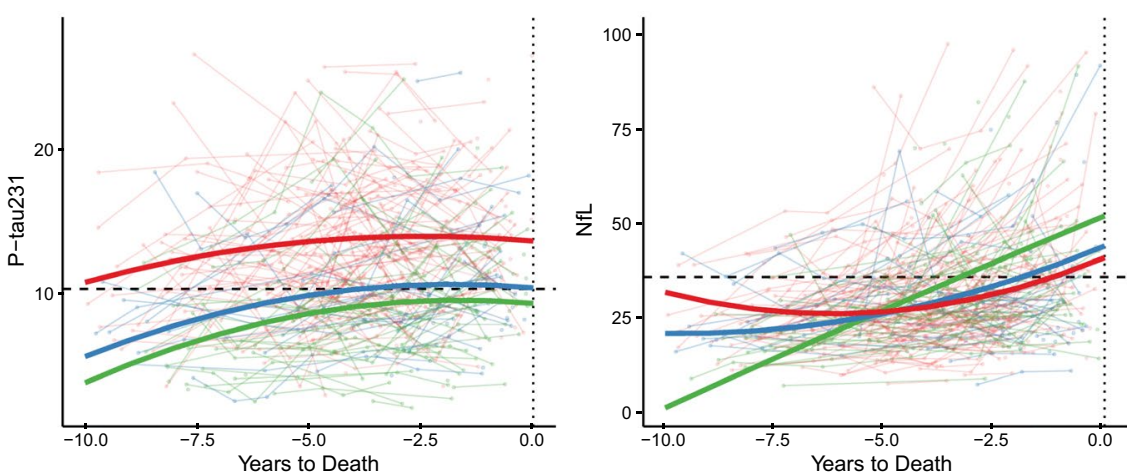

Fig. 5 Longitudinal changes of plasma biomarkers in relation to ADNC. Longitudinal progression in biomarkers in the 10 years prior to death in all study participants were divided by their degree of ADNC. Horizontal dashed lines represent the thresholds derived from ROC analyses presented in Fig. 4. Thick lines represent predictions of the trajectories of the biomarkers for a demographically average par- ticipant, derived from mixed effects models with covariates added for age, sex, interval from last visit to death, as well as each variable's interaction with time. All models included random intercepts and slopes by participant. A version of this figure and analysis excluding individuals with concomitant non-AD pathologies is available in Supplementary Fig. 2, On-line Resource 


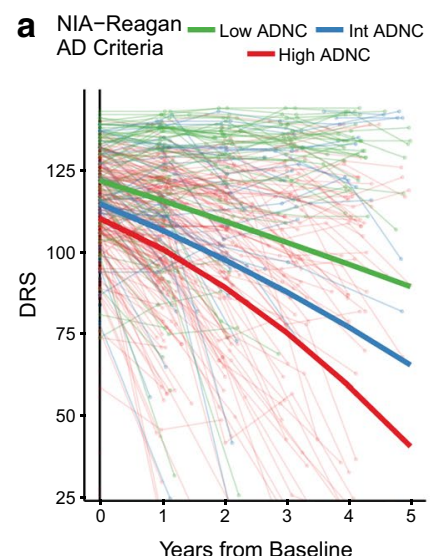

b $\quad-\mathrm{P}-\operatorname{tau} 181<3.96-\mathrm{P}-\operatorname{tau} 181>3.96$

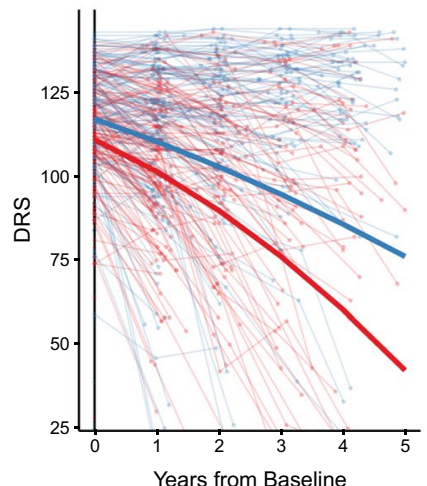

Fig. 6 Plasma P-tau and NfL biomarkers and longitudinal cognitive change in relation to AD pathology (excluding FTLD, HS, LBD, and Other pathologies). Longitudinal progression on the Dementia Rating Scale (DRS) in the 5-year interval from baseline in study participants divided by their a degree of ADNC, or $\mathbf{b}-\mathbf{d}$ baseline plasma biomarker levels, after excluding FTLD, HS, LBD, and other significant pathologies. Cutoffs for each biomarker are those derived from ROC analyses presented in Fig. 4. Thick lines represent predictions of the trajectories of the DRS for a demographically average partic-
C $-\mathrm{P}$-tau231 $<10.48$ - $\mathrm{P}$-tau231 $>10.48$ d $-\mathrm{NfL}<36.5$ - NfL $>36.5$
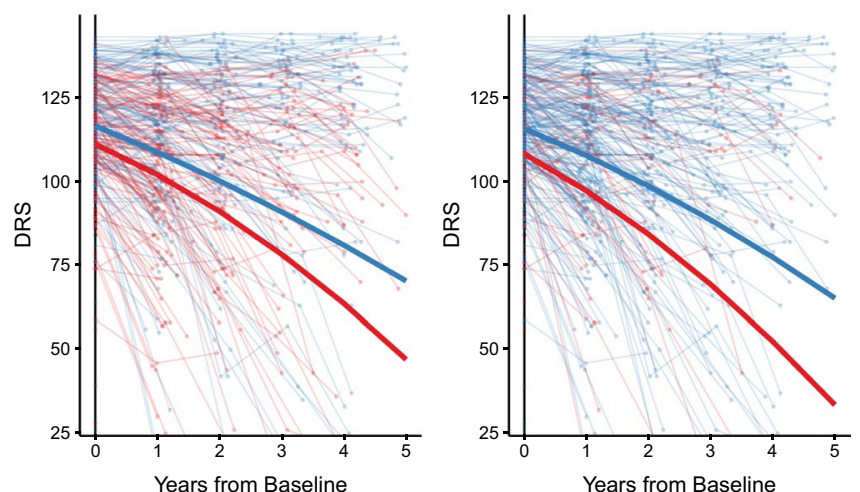

ipant, derived from mixed effects models with covariates added for age, sex, interval from last visit to death, education as well as each variable's interaction with time. To account for different starting levels of impairment the baseline DRS score was included as an interaction with time. All models included random intercepts and slopes by participant. Statistics for Exponential time term by biomarker interaction: NIA-Reagan Low vs Int, $p=0.24$, Low vs High $p=9.4 \times 10^{-12}$, pTau181 $p=6.6 \times 10^{-7}$, pTau231 $p=0.0022$, NfL $p=0.021$
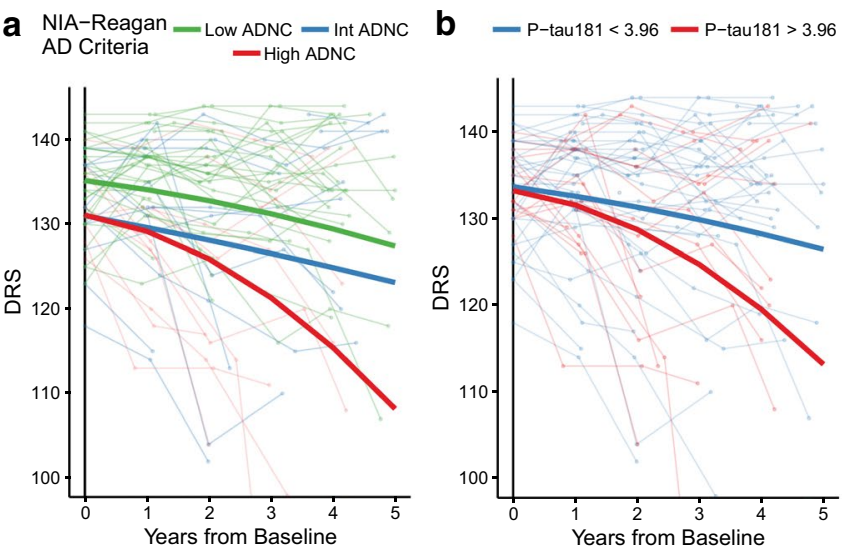

Fig. 7 ADNC and baseline plasma P-tau and NfL biomarkers and longitudinal cognitive change in subjects with normal cognition or MCI at baseline. Longitudinal progression on the Dementia Rating Scale (DRS) in the 5-year interval from baseline in participants with normal cognition of mild cognitive impairment (MCI) divided by their a degree of ADNC, or b-d baseline plasma biomarker levels, after excluding FTLD, HS, LBD, and other significant pathologies. Cutoffs for each biomarker are those derived from ROC analyses presented in Fig. 4. Thick lines represent predictions of the trajectories
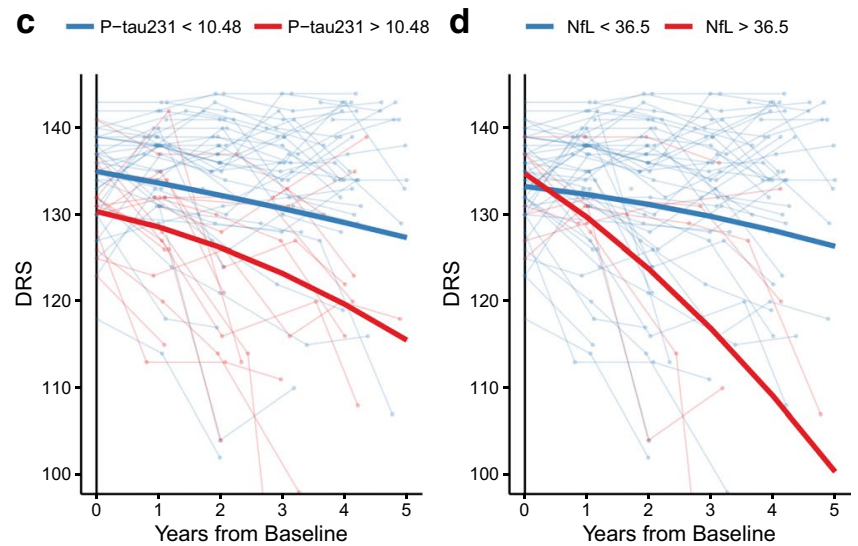

of the DRS for a demographically average participant, derived from mixed effects models with covariates added for age, sex, interval from last visit to death, education as well as each variable's interaction with time. To account for different starting levels of impairment the baseline DRS score was included as an interaction with time. All models included random intercepts and slopes by participant. Statistics for exponential time term by biomarker interaction: NIA-Reagan Low vs Int, $p=0.66$, Low vs High $p=0.0074$, pTau181 $p=0.0019$, pTau231 $p=0.044$, NfL $p=0.00524$ 
Table 3 Vascular risk factors and neuropathological changes in the high and low plasma NfL subgroups

\begin{tabular}{llll}
\hline & NfL $<36.5$ & NfL $>36.5$ & $p$ value \\
\hline Number of participants & 185 & 127 & \\
Last age & $78.8 \pm 9.4$ & $82 \pm 8.5$ & $\mathbf{0 . 0 0 2}$ \\
Age at death & $81.1 \pm 9.3$ & $84 \pm 8.5$ & $\mathbf{0 . 0 0 5}$ \\
Smoking ever & $38(21 \%)$ & $35(28 \%)$ & 0.18 \\
History of cardiovascular disease & $66(36 \%)$ & $54(43 \%)$ & 0.27 \\
Atrial fibrillation & $22(12 \%)$ & $29(23 \%)$ & $\mathbf{0 . 0 2}$ \\
Diabetes mellitus & $14(8 \%)$ & $9(7 \%)$ & 0.99 \\
Hypertension & $94(51 \%)$ & $78(61 \%)$ & 0.08 \\
History of TIA or stroke & $26(14 \%)$ & $29(23 \%)$ & $\mathbf{0 . 0 4}$ \\
Pathology: cerebral infarct & $16(9 \%)$ & $13(10 \%)$ & 0.78 \\
Pathology: microinfarcts & $7(4 \%)$ & $22(17 \%)$ & $\mathbf{0 . 0 0 0 1}$ \\
Pathology: atherosclerosis (mod- & $58(31 \%)$ & $65(51 \%)$ & $\mathbf{0 . 0 0 0 4}$ \\
erate or severe) & & & \\
\hline
\end{tabular}

$p<0.05$ in bold

Statistical comparisons by $t$-test for continuous variables and Chisquared test for binary variables (presence/absence of risk factor or pathology)

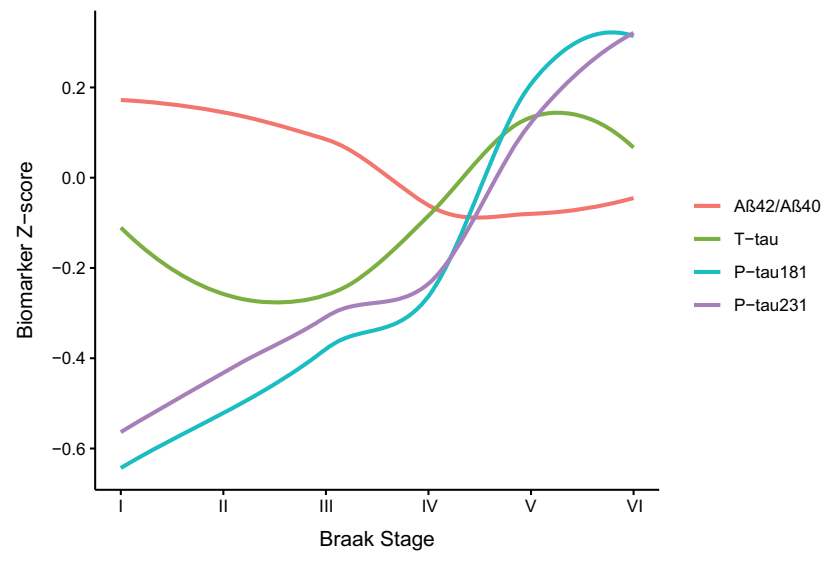

Fig. 8 Plasma biomarker Z-scores and Braak stage. Local regression curves derived by locally estimated scatterplot smoothing of the $Z$-score of each plasma biomarker from the blood draw closest to death across Braak stage. The individual biomarker $Z$-scores were derived by setting the mean of the distribution to 0 and its standard deviation to 1

Supplementary Information The online version contains supplementary material available at https://doi.org/10.1007/s00401-022-02408-5.

Acknowledgements $\mathrm{HZ}$ is a Wallenberg Scholar supported by grants from the Swedish Research Council (\#2018-02532), the European Research Council (\#681712), Swedish State Support for Clinical Research (\#ALFGBG-720931), the Alzheimer Drug Discovery Foundation (ADDF), USA (\#201809-2016862), the AD Strategic Fund and the Alzheimer's Association (\#ADSF-21-831376-C, \#ADSF-21831381-C and \#ADSF-21-831377-C), the Olav Thon Foundation, the Erling-Persson Family Foundation, Stiftelsen för Gamla Tjänarinnor, Hjärnfonden, Sweden (\#FO2019-0228), the European Union's Horizon 2020 research and innovation programme under the Marie
Skłodowska-Curie grant agreement No 860197 (MIRIADE), and the UK Dementia Research Institute at UCL. KB is supported by the Swedish Research Council (\#2017-00915), the Alzheimer Drug Discovery Foundation (ADDF), USA (\#RDAPB-201809-2016615), the Swedish Alzheimer Foundation (\#AF-742881), Hjärnfonden, Sweden (\#FO2017-0243), the Swedish state under the agreement between the Swedish government and the County Councils, the ALF-agreement (\#ALFGBG-715986), and European Union Joint Program for Neurodegenerative Disorders (JPND2019-466-236). DG, DPS, AH and RAR are supported by funding to the Shiley-Marcos ADRC at UC San Diego (NIH/NIA P30-AG062429). DSS is supported by National Institute on Aging grant F30-AG063440.

\section{Declarations}

Conflict of interest HZ has served at scientific advisory boards and/or as a consultant for Abbvie, Alector, Eisai, Denali, Roche Diagnostics, Wave, Samumed, Siemens Healthineers, Pinteon Therapeutics, Nervgen, AZTherapies, CogRx, and Red Abbey Labs, has given lectures in symposia sponsored by Cellectricon, Fujirebio, Alzecure and Biogen, and is a co-founder of Brain Biomarker Solutions in Gothenburg $A B$ (BBS), which is a part of the GU Ventures Incubator Program (outside submitted work). KB has served as a consultant, at advisory boards, or at data monitoring committees for Abcam, Axon, Biogen, Julius Clinical, Lilly, MagQu, Novartis, Roche Diagnostics, and Siemens Healthineers, and is a co-founder of Brain Biomarker Solutions in Gothenburg AB (BBS), which is a part of the GU Ventures Incubator Program (outside submitted work). DPS has served as a consultant for Biogen and Aptinyx. DG has served as a consultant for Biogen, Roche, General Electric Healthcare, Fujirebio, Amprion, Generian and Cognition Therapeutics.

Open Access This article is licensed under a Creative Commons Attribution 4.0 International License, which permits use, sharing, adaptation, distribution and reproduction in any medium or format, as long as you give appropriate credit to the original author(s) and the source, provide a link to the Creative Commons licence, and indicate if changes were made. The images or other third party material in this article are included in the article's Creative Commons licence, unless indicated otherwise in a credit line to the material. If material is not included in the article's Creative Commons licence and your intended use is not permitted by statutory regulation or exceeds the permitted use, you will need to obtain permission directly from the copyright holder. To view a copy of this licence, visit http://creativecommons.org/licenses/by/4.0/.

\section{References}

1. Ashton NJ, Janelidze S, Al Khleifat A, Leuzy A, van der Ende EL, Karikari TK et al (2021) A multicentre validation study of the diagnostic value of plasma neurofilament light. Nat Commun 12:3400. https://doi.org/10.1038/s41467-021-23620-z

2. Ashton NJ, Leuzy A, Lim YM, Troakes C, Hortobágyi T, Höglund $K$ et al (2019) Increased plasma neurofilament light chain concentration correlates with severity of post-mortem neurofibrillary tangle pathology and neurodegeneration. Acta Neuropathol Commun 7:5. https://doi.org/10.1186/s40478-018-0649-3

3. Ashton NJ, Pascoal TA, Karikari TK, Benedet AL, Lantero-Rodriguez J, Brinkmalm G et al (2021) Plasma p-tau231: a new biomarker for incipient Alzheimer's Disease pathology. Acta Neuropathol 141:709-724. https://doi.org/10.1007/s00401-021-02275-6

4. Bangen KJ, Thomas KR, Weigand AJ, Edmonds EC, Clark AL, Solders S et al (2021) Elevated plasma neurofilament light predicts a faster rate of cognitive decline over 5 years in participants 
with objectively-defined subtle cognitive decline and MCI. Alzheimer's Dementia alz. https://doi.org/10.1002/alz.12324

5. Boyle PA, Yang J, Yu L, Leurgans SE, Capuano AW, Schneider JA et al (2017) Varied effects of age-related neuropathologies on the trajectory of late life cognitive decline. Brain 140:804-812. https://doi.org/10.1093/brain/aww341

6. Braak H, Alafuzoff I, Arzberger T, Kretzschmar H, Del Tredici K (2006) Staging of Alzheimer disease-associated neurofibrillary pathology using paraffin sections and immunocytochemistry. Acta Neuropathol 112:389-404. https://doi.org/10.1007/ s00401-006-0127-z

7. Brickman AM, Manly JJ, Honig LS, Sanchez D, Reyes-Dumeyer D, Lantigua RA et al (2021) Plasma p-tau181, p-tau217, and other blood-based Alzheimer's Disease biomarkers in a multi-ethnic, community study. Alzheimer's Dementia alz. https://doi.org/10. 1002/alz.12301

8. Cullen NC, Leuzy A, Janelidze S, Palmqvist S, Svenningsson AL, Stomrud E et al (2021) Plasma biomarkers of Alzheimer's disease improve prediction of cognitive decline in cognitively unimpaired elderly populations. Nat Commun 12:3555. https://doi.org/10. 1038/s41467-021-23746-0

9. Galasko D, Xiao M, Xu D, Smirnov D, Salmon DP, Dewit $\mathrm{N}$ et al (2019) Synaptic biomarkers in CSF aid in diagnosis, correlate with cognition and predict progression in MCI and Alzheimer's Disease. Alzheimers Dement (NY) 5:871-882. https://doi.org/10. 1016/j.trci.2019.11.002

10. Groveman BR, Orrù CD, Hughson AG, Raymond LD, Zanusso G, Ghetti B et al (2018) Rapid and ultra-sensitive quantitation of disease-associated $\alpha$-synuclein seeds in brain and cerebrospinal fluid by $\alpha$ Syn RT-QuIC. Acta Neuropathol Commun 6:7. https:// doi.org/10.1186/s40478-018-0508-2

11. Guo T, Shaw LM, Trojanowski JQ, Jagust WJ, Landau SM, Alzheimer's Disease Neuroimaging Initiative (2020) Association of CSF A $\beta$, amyloid PET, and cognition in cognitively unimpaired elderly adults. Neurology 95:e2075-e2085. https://doi.org/10. 1212/WNL.0000000000010596

12. Hampel H, Cummings J, Blennow K, Gao P, Jack CR, Vergallo A (2021) Developing the ATX(N) classification for use across the Alzheimer disease continuum. Nat Rev Neurol 17:580-589. https://doi.org/10.1038/s41582-021-00520-w

13. Hyman BT, Trojanowski JQ (1997) Consensus recommendations for the postmortem diagnosis of Alzheimer disease from the National Institute on Aging and the Reagan Institute Working Group on diagnostic criteria for the neuropathological assessment of Alzheimer disease. J Neuropathol Exp Neurol 56:1095-1097. https://doi.org/10.1097/00005072-199710000-00002

14. Ikonomovic MD, Buckley CJ, Abrahamson EE, Kofler JK, Mathis CA, Klunk WE et al (2020) Post-mortem analyses of PiB and flutemetamol in diffuse and cored amyloid- $\beta$ plaques in Alzheimer's Disease. Acta Neuropathol 140:463-476. https://doi.org/10. 1007/s00401-020-02175-1

15. Jack CR, Bennett DA, Blennow K, Carrillo MC, Dunn B, Haeberlein SB et al (2018) NIA-AA Research Framework: toward a biological definition of Alzheimer's Disease. Alzheimers Dement 14:535-562. https://doi.org/10.1016/j.jalz.2018.02.018

16. Karanth S, Nelson PT, Katsumata Y, Kryscio RJ, Schmitt FA, Fardo DW et al (2020) Prevalence and clinical phenotype of quadruple misfolded proteins in older adults. JAMA Neurol 77:12991307. https://doi.org/10.1001/jamaneurol.2020.1741

17. Karikari TK, Pascoal TA, Ashton NJ, Janelidze S, Benedet AL, Rodriguez JL et al (2020) Blood phosphorylated tau 181 as a biomarker for Alzheimer's Disease: a diagnostic performance and prediction modelling study using data from four prospective cohorts. Lancet Neurol 19:422-433. https://doi.org/10.1016/ S1474-4422(20)30071-5
18. Keshavan A, Pannee J, Karikari TK, Rodriguez JL, Ashton NJ, Nicholas JM et al (2021) Population-based blood screening for preclinical Alzheimer's Disease in a British birth cohort at age 70. Brain 144(2):434-449. https://doi.org/10.1093/brain/awaa403

19. Keshavan A, Pannee J, Karikari TK, Rodriguez JL, Ashton NJ, Nicholas JM et al (2021) Population-based blood screening for preclinical Alzheimer's Disease in a British birth cohort at age 70. Brain 144:434-449

20. Lantero Rodriguez J, Karikari TK, Suárez-Calvet M, Troakes C, King A, Emersic A et al (2020) Plasma p-tau181 accurately predicts Alzheimer's Disease pathology at least 8 years prior to post-mortem and improves the clinical characterisation of cognitive decline. Acta Neuropathol 140:267-278. https://doi.org/10. 1007/s00401-020-02195-x

21. Leuzy A, Janelidze S, Mattsson-Carlgren N, Palmqvist S, Jacobs D, Cicognola C et al (2021) Comparing the clinical utility and diagnostic performance of cerebrospinal Fluid P-Tau181, P-Tau217 and P-Tau231 assays. Neurology. https://doi.org/10. 1212/WNL.0000000000012727

22. Leverenz JB, Hamilton R, Tsuang DW, Schantz A, Vavrek D, Larson EB et al (2008) Empiric refinement of the pathologic assessment of Lewy-related pathology in the dementia patient. Brain Pathol 18:220-224. https://doi.org/10.1111/j.1750-3639.2007. 00117.x

23. Luo J, Agboola F, Grant E, Masters CL, Albert MS, Johnson SC et al (2020) Sequence of Alzheimer Disease biomarker changes in cognitively normal adults: a cross-sectional study. Neurology 95:e3104-e3116. https://doi.org/10.1212/WNL.0000000000 010747

24. Mammana A, Baiardi S, Quadalti C, Rossi M, Donadio V, Capellari S et al (2021) RT-QuIC detection of pathological $\alpha$-synuclein in skin punches of patients with lewy body disease. Mov Disord. https://doi.org/10.1002/mds.28651

25. Mielke MM, Frank RD, Dage JL, Jeromin A, Ashton NJ, Blennow $\mathrm{K}$ et al (2021) Comparison of plasma phosphorylated tau species with amyloid and tau positron emission tomography, neurodegeneration, vascular pathology, and cognitive outcomes. JAMA Neurol. https://doi.org/10.1001/jamaneurol.2021.2293

26. Mirra SS, Heyman A, McKeel D, Sumi SM, Crain BJ, Brownlee LM et al (1991) The consortium to establish a registry for Alzheimer's Disease (CERAD). Part II. Standardization of the neuropathologic assessment of Alzheimer's Disease. Neurology 41:479-486. https://doi.org/10.1212/wnl.41.4.479

27. Montine TJ, Phelps CH, Beach TG, Bigio EH, Cairns NJ, Dickson DW et al (2012) National Institute on Aging-Alzheimer's Association guidelines for the neuropathologic assessment of Alzheimer's Disease: a practical approach. Acta Neuropathol 123:1-11. https:// doi.org/10.1007/s00401-011-0910-3

28. Nakamura A, Kaneko N, Villemagne VL, Kato T, Doecke J, Doré $\mathrm{V}$ et al (2018) High performance plasma amyloid- $\beta$ biomarkers for Alzheimer's Disease. Nature 554:249-254. https://doi.org/10. 1038/nature25456

29. Nelson PT, Dickson DW, Trojanowski JQ, Jack CR, Boyle PA, Arfanakis K et al (2019) Limbic-predominant age-related TDP-43 encephalopathy (LATE): consensus working group report. Brain 142:1503-1527. https://doi.org/10.1093/brain/awz099

30. Ossenkoppele R, Reimand J, Smith R, Leuzy A, Strandberg O, Palmqvist S et al (2021) Tau PET correlates with different Alzheimer's Disease-related features compared to CSF and plasma p-tau biomarkers. EMBO Mol Med 13:e14398. https://doi.org/10. 15252/emmm.202114398

31. Palmqvist S, Janelidze S, Quiroz YT, Zetterberg H, Lopera F, Stomrud E et al (2020) Discriminative accuracy of plasma phospho-tau 217 for Alzheimer Disease vs other neurodegenerative disorders. JAMA 324:772. https://doi.org/10.1001/jama.2020. 12134 
32. Palmqvist S, Mattsson N, Hansson O, Alzheimer's Disease Neuroimaging Initiative (2016) Cerebrospinal fluid analysis detects cerebral amyloid- $\beta$ accumulation earlier than positron emission tomography. Brain 139:1226-1236. https://doi.org/10.1093/brain/ aww015

33. Palmqvist $\mathrm{S}$, Tideman $\mathrm{P}$, Cullen N, Zetterberg H, Blennow K, Alzheimer's Disease Neuroimaging Initiative et al (2021) Prediction of future Alzheimer's Disease dementia using plasma phospho-tau combined with other accessible measures. Nat Med 27:1034-1042. https://doi.org/10.1038/s41591-021-01348-Z

34. Rossi M, Baiardi S, Teunissen CE, Quadalti C, van de Beek M, Mammana A et al (2021) Diagnostic value of the CSF $\alpha$-synuclein real-time quaking-induced conversion assay at the prodromal MCI stage of dementia with Lewy bodies. Neurology. https://doi.org/ 10.1212/WNL.0000000000012438

35. Schindler SE, Bollinger JG, Ovod V, Mawuenyega KG, Li Y, Gordon BA et al (2019) High-precision plasma $\beta$-amyloid 42/40 predicts current and future brain amyloidosis. Neurology 93:e1647e1659. https://doi.org/10.1212/WNL.0000000000008081

36. Smirnov DS, Galasko D, Hansen LA, Edland SD, Brewer JB, Salmon DP (2019) Trajectories of cognitive decline differ in hippocampal sclerosis and Alzheimer's Disease. Neurobiol Aging 75:169-177. https://doi.org/10.1016/j.neurobiolaging.2018.11. 015

37. Suárez-Calvet M, Karikari TK, Ashton NJ, Lantero Rodríguez J, Milà-Alomà M, Gispert JD et al (2020) Novel tau biomarkers phosphorylated at T181, T217 or T231 rise in the initial stages of the preclinical Alzheimer's continuum when only subtle changes in $\mathrm{A} \beta$ pathology are detected. EMBO Mol Med 12:e12921. https:// doi.org/10.15252/emmm.202012921

38. Thal DR, Rüb U, Schultz C, Sassin I, Ghebremedhin E, Del Tredici $\mathrm{K}$ et al (2000) Sequence of A $\beta$-protein deposition in the human medial temporal lobe. J Neuropathol Exp Neurol 59:733-748. https://doi.org/10.1093/jnen/59.8.733

39. Uchikado H, Lin W-L, DeLucia MW, Dickson DW (2006) Alzheimer Disease with amygdala Lewy bodies: a distinct form of alpha-synucleinopathy. J Neuropathol Exp Neurol 65:685-697. https://doi.org/10.1097/01.jnen.0000225908.90052.07

40. West T, Kirmess KM, Meyer MR, Holubasch MS, Knapik SS, $\mathrm{Hu} \mathrm{Y}$ et al (2021) A blood-based diagnostic test incorporating plasma A $\beta 42 / 40$ ratio, ApoE proteotype, and age accurately identifies brain amyloid status: findings from a multi cohort validity analysis. Mol Neurodegeneration 16:30. https://doi.org/10.1186/ s13024-021-00451-6

41. Wharton SB, Wang D, Parikh C, Matthews FE, Brayne C, Ince PG et al (2019) Epidemiological pathology of $A \beta$ deposition in the ageing brain in CFAS: addition of multiple $A \beta$-derived measures does not improve dementia assessment using logistic regression and machine learning approaches. Acta Neuropathol Commun 7:198. https://doi.org/10.1186/s40478-019-0858-4

Publisher's Note Springer Nature remains neutral with regard to jurisdictional claims in published maps and institutional affiliations.

\section{Authors and Affiliations}

\section{Denis S. Smirnov' ${ }^{1}$ Nicholas J. Ashton ${ }^{2,3,4,5} \cdot$ Kaj Blennow $^{2,6} \cdot$ Henrik Zetterberg ${ }^{2,6,7,8,9}$. Joel Simrén ${ }^{2,6}$. Juan Lantero-Rodriguez ${ }^{2}$. Thomas K. Karikari ${ }^{2,10}$ • Annie Hiniker ${ }^{1} \cdot$ Robert A. Rissman $^{1}$ • David P. Salmon ${ }^{1}$. Douglas Galasko ${ }^{1,11}$ [1]}

1 University of California, San Diego and Shiley-Marcos Alzheimer's Disease Research Center, La Jolla, CA, USA

2 Institute of Neuroscience and Physiology, Department of Psychiatry and Neurochemistry, Sahlgrenska Academy, University of Gothenburg, Mölndal, Sweden

3 Institute of Psychiatry, Psychology and Neuroscience, King's College London, Maurice Wohl Clinical Neuroscience Institute, London, UK

4 NIHR Biomedical Research Centre for Mental Health and Biomedical Research Unit for Dementia at South London and Maudsley NHS Foundation, London, UK

5 Wallenberg Centre for Molecular and Translational Medicine, Department of Psychiatry and Neurochemistry, Institute of Neuroscience and Physiology, Sahlgrenska Academy at the University of Gothenburg, Gothenburg, Sweden
6 Clinical Neurochemistry Laboratory, Sahlgrenska University Hospital, Mölndal, Sweden

7 Department of Neurodegenerative Disease, UCL Institute of Neurology, Queen Square, London, UK

8 UK Dementia Research Institute, London, UK

9 Hong Kong Center for Neurodegenerative Diseases, Hong Kong, China

10 Department of Psychiatry, University of Pittsburgh, Pittsburgh, PA, USA

11 Department of Neurosciences, UC San Diego, 9500 Gilman Drive, La Jolla, CA 92093-0624, USA 